\title{
Stream Sampling Framework and Application for Frequency Cap Statistics
}

\author{
EDITH COHEN, Google AI, CA, USA and Tel Aviv University, Israel
}

\begin{abstract}
Unaggregated data, in a streamed or distributed form, are prevalent and come from diverse sources such as interactions of users with web services and IP traffic. Data elements have keys (cookies, users, queries), and elements with different keys interleave. Analytics on such data typically utilizes statistics expressed as a sum over keys in a specified segment of a function $f$ applied to the frequency (the total number of occurrences) of the key. In particular, Distinct is the number of active keys in the segment, Sum is the sum of their frequencies, and both are special cases of frequency cap statistics, which cap the frequency by a parameter $T$.

Random samples can be very effective for quick and efficient estimation of statistics at query time. Ideally, to estimate statistics for a given function $f$, our sample would include a key with frequency $w$ with probability roughly proportional to $f(w)$. The challenge is that while such "gold-standard" samples can be easily computed after aggregating the data (computing the set of key-frequency pairs), this aggregation is costly: It requires structure of size that is proportional to the number of active keys, which can be very large.

We present a sampling framework for unaggregated data that uses a single pass (for streams) or two passes (for distributed data) and structure size proportional to the desired sample size. Our design unifies classic solutions for Distinct and Sum. Specifically, our $\ell$-capped samples provide nonnegative unbiased estimates of any monotone non-decreasing frequency statistics and statistical guarantees on quality that are close to gold standard for cap statistics with $T=\Theta(\ell)$. Furthermore, our multi-objective samples provide these statistical guarantees on quality for all concave sub-linear statistics (the nonnegative span of cap functions) while incurring only a logarithmic overhead on sample size.
\end{abstract}

\section{CCS Concepts: • Theory of computation $\rightarrow$ Sketching and sampling;}

Additional Key Words and Phrases: Frequency statistics, distributed aggregation, stream processing

ACM Reference format:

Edith Cohen. 2018. Stream Sampling Framework and Application for Frequency Cap Statistics. ACM Trans. Algorithms 14, 4, Article 52 (September 2018), 40 pages.

https://doi.org/10.1145/3234338

\section{INTRODUCTION}

The data available from many services, such as interactions of users with Web services or content, search logs, and IP traffic, are presented in an unaggregated form. ${ }^{1}$ In this model, each data element $e=(e . k e y, e . v a l)$ has a key from a universe $\mathcal{X}$ and a value e.val $>0$. Multiple elements can have the same key and data elements are streamed or reside in distributed storage.

\footnotetext{
${ }^{1}$ Preliminary version of the work appeared in ACM KDD 2015 [8].
}

Authors' addresses: E. Cohen, 1600 Amphitheatre Pkwy, Mountain View, CA 94043, USA; email: edith@cohenwang.com. Permission to make digital or hard copies of all or part of this work for personal or classroom use is granted without fee provided that copies are not made or distributed for profit or commercial advantage and that copies bear this notice and the full citation on the first page. Copyrights for components of this work owned by others than ACM must be honored. Abstracting with credit is permitted. To copy otherwise, or republish, to post on servers or to redistribute to lists, requires prior specific permission and/or a fee. Request permissions from permissions@acm.org.

(C) 2018 Association for Computing Machinery.

1549-6325/2018/09-ART52 \$15.00

https://doi.org/10.1145/3234338 
The aggregated view of the data is a set of key value pairs $\left\{\left(x, w_{x}\right)\right\}$ where each active key $x \in \mathcal{X}$ (a key that occurred in at least one data element) has a unique element and $w_{x}=\sum_{e \mid e . k e y=x}$ e.val (the sum of the values of all data elements with key $x$ ). When element are unweighted (have uniform values - that is, all elements $e$ have $e . v a l=1), w_{x}$ is the number of data elements with key $x$ and is often referred to as the frequency of the key $x$ (it is proportional to the actual frequency in the dataset). For consistency, we will refer to $w_{x}$ as frequency also with weighted element values.

Frequency statistics of such data are fundamental to data analytics. Queries have the form

$$
Q(f, H) \equiv \sum_{x \in \mathcal{X} \cap H} f\left(w_{x}\right)
$$

where $f(w) \geq 0$ is a nonnegative function such that $f(0)=0$ and $H$ is a selection predicate that specifies a segment of the key population $\mathcal{X}$. Typically $f$ is monotone non-decreasing, which means that more frequent keys carry at least the same contribution as less frequent ones. Some prominent examples are the $p$ th frequency moment, where $f(x)=x^{p}$ for $p>0$ [1] and frequency cap statistics, where $f$ is a cap function with parameter $T>0$ :

$$
\operatorname{cap}_{T}(y) \equiv \min \{y, T\} .
$$

Two special cases of both cap statistics and frequency moments, that are widely studied and applied in big data analytics, are Distinct, the number of distinct (active) keys in the segment $\left(L_{0}\right.$ moment or $\mathrm{cap}_{1}$, assuming elements values are $\geq 1$ ), and Sum, the sum of values of elements with keys in the segment $\left(L_{1}\right.$ moment or cap $\left.{ }_{\infty}\right)$.

General cap functions are between these extremes and are used to mitigate the domination of the statistics by the (typically few) very frequent keys while still providing a larger representation of the more frequent keys. Cap statistics are prevalent in online advertising platforms [24, 35]: A common practice is to allow an advertiser to specify a limit to the number of impressions of an ad campaign that any individual user is exposed to in a particular duration of time. Advertisements also typically target only a segment $H$ of users (say, certain demographics and geographic location). The statistics $Q\left(\operatorname{cap}_{T}, H\right)$ is the number of qualifying opportunities for placing an ad. These queries are posed over past data to provide an advertiser with a prediction for the total potential number of qualifying impressions. Often, the prediction needs to be computed or estimated quickly to facilitate interactive campaign planning.

Computation of exact frequency statistics (1) requires aggregating the data by key. The representation size of the aggregated view, however, and the runtime structure size needed to produce it, are linear in the number of distinct keys. Often, the number of distinct keys is very large, whereas scalable computation limits us to use one or few passes over elements and a small runtime structure (which translates to memory or communication). With streamed data that are discarded (such as with IP traffic), we are limited to a single pass. These constraints motivated the study of small summaries of the dataset that provide approximate answers [1, 21, 23].

Random samples are effective summaries of the data. We can sample the data by first computing the aggregated view of key value pairs $\left\{\left(x, w_{x}\right)\right\}$, for each key $x$ computing a weight $f\left(w_{x}\right)$, and we then computing a weighted sample of the data. Some classic schemes include Probability Proportion to Size (pps) [39], VarOpt $[4,13]$, or bottom- $k$, which includes successive weighted sampling without replacement (ppswor) and sequential Poisson/Priority sampling [17, 34, 36, 37].

From such weighted samples, we can compute approximate segment frequency statistics by applying an appropriate estimator to the sample. There is a well-understood tradeoff between the sample size $k$ and the statistical guarantees on the quality of our approximation. For a segment $H$ that has proportion $q=Q(f, H) / Q(f, \mathcal{X})$ of the statistics value on the general key population, there is an upper bound on the coefficient of variation $(\mathrm{CV})$ of (roughly) $(q k)^{-0.5}$ : the inverse of 
the square root of $q k$. The CV is the standard error normalized by the mean, and (for unbiased estimators) is equal to the normalized root mean square error (NRMSE). That is, to obtain NRMSE of $\epsilon=0.1(10 \%)$ on segments that have at least $q=0.001$ fraction of the total value of the statistics, we need to choose a sample size of $k=\epsilon^{-2} / q=10^{5}$, which is usually much smaller than the number of distinct keys we might have. This also means that we can obtain confidence intervals on our estimates using the actual number of samples from our segment. Moreover, this CV bound is worstcase tight (when considering all segments of same $q$ and distributions that are not dominated by few keys) even for schemes applied to the aggregated data and will be the gold standard we hold ourselves against when designing sampling schemes and estimators.

Our challenge is to design sampling schemes that can be realized using a structure size that is of the order of the desired sample size, perform one or few passes over the data, and achieve tradeoff of size and estimation quality that is close to the aggregated gold standard. There is a large body of work on stream sampling schemes designed for distinct and sum queries. The Sample and Hold (SH) family of sampling schemes [10, 12, 18, 23] and another based on VarOpt [11] are suited for sum queries. Distinct reservoir sampling of keys [21,31] is suited for distinct queries. Both $\mathrm{SH}$ [12] and distinct sampling support unbiased estimates of all frequency statistics and meet our $(q k)^{-0.5} \mathrm{CV}$ upper bound target for the particular statistics they are designed for (the claim for $\mathrm{SH}$ is established here). They do not provide, however, comparable statistical guarantees for other statistics.

\subsection{Contributions and Overview}

We present a sampling framework for unaggregated data that generalizes existing schemes for distinct and $\mathrm{SH}$ sampling and bottom- $k$ sampling of aggregated data. A sampling scheme in our framework is specified by a random scoring function that can be applied in isolation to data elements and is tailored to the statistics we want to estimate. We accordingly define the seed value of a key, which is also a random variable, to be the minimum score of an element with this key. Finally, our sample includes a set of keys with smallest seed values. Our framework is detailed in Section 3.

In Section 4, we present a two-pass algorithm that identifies the sampled keys in the first pass and computes their frequencies in a second pass to facilitate estimation. The summary structures are composable, which allows for parallel or distributed computation. Our streaming schemes have the same sample distribution as the two-pass schemes, but collect only "partial information" $c_{x} \leq w_{x}$ on the frequency $w_{x}$ of each sampled key $x$. These partial frequencies is what we use for estimation. In Section 5, we present a generic one-pass algorithm that applies only with unweighted elements, and in Section 6 we cast distinct and classic SH sampling schemes as special cases.

We then present sampling schemes that are tailored for general frequency cap statistics. We use two basic designs of element scoring functions: a more intuitive "discrete" design that only applies to uniform-value elements and a more powerful "continuous" design that applies with arbitrary positive element values. Both designs generalize the element scoring function used for distinct and classic SH sampling. In Section 7, we specify our discrete-design schemes, d-SH $\mathrm{H}_{\ell}$, which are parametrized by an integer $\ell$ (the cap parameter). When $\ell$ is larger than the maximum frequency over keys, the scheme d-SH $\mathrm{SH}_{\ell}$ approaches classic $\mathrm{SH}$ and when $\ell=1$, it is identical to distinct sampling. We express the unique unbiased and admissible estimators for all $\ell$ and any discrete frequency statistics, that is, $f$ specified for nonnegative integers.

In Section 8, we present our continuous-design schemes $\mathrm{SH}_{\ell}$, where the parameter $\ell$ is a positive real value. The exposition does not rely on the discrete design, so this section can be read after Section 3. When $\ell \gg \max _{x} w_{x}, \mathrm{SH}_{\ell}$ approaches weighted sampling by $w_{x}$ (a weighted version of 
Sample and Hold) [10]. For $\ell \ll \min _{x} w_{x}, \mathrm{SH}_{\ell}$ approaches distinct sampling. We derive estimators of frequency statistics where the function $f$ is continuous and differentiable almost everywhere. Note that most natural statistics, including frequency moments and cap statistics, can be expressed as continuous monotone functions, which are differentiable almost everywhere. Surprisingly perhaps, the continuous design yields an elegant, closed-form, and simple specification of estimators.

We show that our estimates of $\mathrm{cap}_{T}$ statistics from $\mathrm{SH}_{\ell}$ samples have CV upper bounded by $O\left((q k)^{-0.5}\right)$ when $T=O(\ell)$. The CV bound is close to the gold standard for the statistics the sample was tailored for and gracefully degrades with disparity $\max \{T / \ell, \ell / T\}$ between the sample cap parameter $\ell$ and the statistics cap parameter $T$. The estimate of any frequency function $f$ from any $\mathrm{SH}_{\ell}$ sample is unbiased and for $f$ that is monotone non-decreasing, also nonnegative. This makes our design very versatile.

Our estimators are derived by expressing sampling as a transform from frequencies to expected "sampled frequencies," and then inverting the transform. The transform is a matrix vector product in the discrete case and an integral transform in the continuous case. For the latter, the estimator is a simple expression in terms of $f$ and its derivative $f^{\prime}$. Since our estimators are the unique inverse of the transform, they are the minimum variance unbiased nonnegative estimators for the sampling scheme, meaning that in terms of variance, they optimally use the information in the sample. Our discrete estimators generalize a matrix inversion applied in References [12,26] to estimate the flow size distribution from Sampled Netflow and SH IP flow records. Our continuous schemes estimators are novel even for the basic weighted SH scheme, for which previously only estimators for sum statistics were provided [10].

In Section 9, we address applications that require estimates with statistical guarantees for multiple, possibly all, concave sublinear statistics. Concave sublinear statistics include all cap func- $_{T}$ tions and all statistics in their nonnegative span $f \in \overline{\text { cap }}[7,9]$ (functions that can be expressed as nonnegative linear combinations of cap functions). This rich and important class includes low frequency moments $\left(f(w)=w^{p}\right.$ for $\left.p \in[0,1]\right)$ and $\log (1+w)$.

One solution is to compute a set of $\mathrm{SH}_{\ell}$ samples with exponentially increasing cap parameter $\ell$. A $\operatorname{cap}_{T}$ statistics query can then be estimated from the sample that has $\ell$ parameter closest to $T$. Any statistics $f$ in the span can be estimated from a respective linear combination of estimates of $\operatorname{cap}_{T}$ statistics. We present a design of a single multi-objective sample that offers both more efficient sampling and a better tradeoff of accuracy and sample size. The design is based on our continuous schemes and draws on a multi-objective schemes for aggregated data [7, 15] based on sample coordination [3]. The multi-objective sample size needed for our target CV bound of $(q k)^{-0.5}$ is at most $k \ln n$ (where $n$ is the number of distinct keys).

Our proposed sampling algorithms and estimators are simple and highly practical, despite the rather technical analysis. The application resembles that of classic $\mathrm{SH}$, distinct sampling, and approximate distinct counting algorithms that are prevalent in industrial applications [25]. Section 10 includes an experimental evaluation that demonstrates superior accuracy versus sample size tradeoffs by using a sample that is suited for the statistic.

\section{PRELIMINARIES}

We work with key-value datasets that consist of elements $e=(e . k e y, e . v a l)$, where e.key is a key from a universe $\mathcal{X}$ and e.val $>0$. The dataset is aggregated if each key appears in at most one element and is unaggregated otherwise. We define the frequency $w_{x} \equiv \sum_{e \mid e . k e y=x}$ e.val of a key $x$ to be the sum of the values of elements with key $x$. If $x$ is not active (there are no elements with key $x$ ), then we define $w_{x}=0$. When element values are uniform, $w_{x}$ is the number of elements with key $x$. The aggregated view of an unaggregated dataset is the set of elements (key value pairs) $\left\{\left(x, w_{x}\right)\right\}$ for all active keys $x$. 
We start with a quick review of relevant sampling schemes for aggregated datasets. A Poisson sample of a key value dataset $\left\{\left(x, w_{x}\right)\right\}$ is specified by sampling probabilities $p_{x}$. The sample $S$ includes each $x \in \mathcal{X}$ with independent probability $p_{x}$ and has expected size $\mathrm{E}[|S|]=\sum_{x} p_{x} \equiv k$. To estimate a frequency statistics $Q(f, H)$ from the sample, we can apply the inverse probability estimator $\overline{Q(f, H)}=\sum_{x \in H \cap S} f\left(w_{x}\right) / p_{x}$ [27]. This estimator can be interpreted as a sum of perkey estimates that are $f\left(w_{x}\right) / p_{x}$ if $x \in S$ and 0 otherwise. Note that this estimator can only be applied when $w_{x}$ and $p_{x}$ are available for all $x \in S$. It is nonnegative and is unbiased if $p_{x}>0$ when $f\left(w_{x}\right)>0$. It is actually the minimum variance unbiased and nonnegative sum estimator (sum of per-key estimates) for the given probabilities $\left\{p_{x}\right\}$.

For data $\left\{\left(x, w_{x}\right)\right\}$, function $f$, and (expected) sample size $k$, one can ask what are the "optimal" sampling probabilities. It is well known that if we sample keys with probability proportional to their contribution $f\left(w_{x}\right)$ (pps), we minimize the sum of per-key variances $\sum_{x} f\left(w_{x}\right)^{2}\left(1 / p_{x}-1\right)$. With pps, we have the following statistical guarantee: For estimates of the statistics $Q(f, H)$, where the segment $H$ has proportion

$$
q=\frac{Q(f, H)}{Q(f, \mathcal{X})}=\frac{\sum_{x \in H} f\left(w_{x}\right)}{\sum_{x} f\left(w_{x}\right)}
$$

of the statistics, the variance of our estimate is

$$
\operatorname{var}[\overline{Q(f, H)}] \leq \frac{1}{q k} Q(f, H)^{2} .
$$

Thus the CV (normalized standard error) is at most $(q k)^{-0.5}$, which is the best bound we can hope for on average over segments with proportion $q$. That is, any scheme that would do better on some segments, would do worse on others. Other weighted sampling schemes we mentioned in the Introduction provide this statistical guarantee with a fixed sample size $k$ : VarOpt provides the $(q k)^{-0.5}$ quality with better estimation for $q$ closer to 1 . Sequential Poisson (Priority) sampling has $(q(k-1))^{-0.5}$ quality [38].

One of these schemes that is particularly relevant for our treatment of unaggregated datasets is ppswor: Keys are selected successively without replacement so that at each step the probability that we select $x$ is proportional to its weight (frequency in unaggregated data) relative to the remaining unsampled keys: $f\left(w_{x}\right) / \sum_{y \notin S} f\left(w_{y}\right)$. The sampling can be realized by associating with each key a random $\operatorname{seed}(x) \sim \operatorname{Exp}\left[f\left(w_{x}\right)\right]$ (exponentially distributed seed with parameter $f\left(w_{x}\right)$ ) The increasing seed value order correspond exactly to ppswor sampling order [36]. A fixed-threshold sample, for a pre-specified threshold $\tau$, includes all keys with seed $(x)<\tau$. Alternatively, we can obtain a fixed size (bottom- $k$ ) sample by taking the $k$ keys with smallest seed values. In the latter case, it is convenient to define $\tau$ as the $(k+1)$ smallest seed.

Finally, we can estimate a statistics $Q(g, H)$ from the ppswor sample taken for weights $f\left(w_{x}\right)$ as follows. When we use fixed threshold sampling, we compute the probability that $x$ is sampled

$$
\Phi_{\tau}\left(w_{x}\right) \equiv \operatorname{Pr}[\operatorname{seed}(x)<\tau]=1-e^{-f\left(w_{x}\right) \tau}
$$

and apply inverse probability:

$$
\widehat{Q(g, H)}=\sum_{x \in H \cap S} \overline{g\left(w_{x}\right)} \mid \tau, \text { where } \overline{g\left(w_{x}\right)} \mid \tau \equiv \frac{g\left(w_{x}\right)}{\Phi_{\tau}\left(w_{x}\right)} .
$$

Note that $\Phi_{\tau}\left(w_{x}\right)$ only depends on $w_{x}$ and $\tau$ (which are available for sampled keys). When we work with a fixed sample size $k$ and define $\tau$ to be the $(k+1)$ smallest seed, we can interpret $\Phi_{\tau}\left(w_{x}\right)$ as the probability that the key $x$ is sampled, conditioned on fixed randomization of other keys. This means that the estimator (2) is unbiased [14]. Moreover, the estimates $\widehat{g\left(w_{x}\right)} \mid \tau$ obtained for different keys $x$ have zero covariances [14], which allows us to bound the variance on segment 
queries using the variance expressions for a pre-specified threshold. In Appendix A, we present self-contained proofs of unbiasedness and nonnegative covariances for bottom- $k$ that extends to the broader framework for unaggregated data that we introduce here. We also outline how we bound $\operatorname{var}[\overline{Q(g, H)}]$ in terms of the per-key fixed-threshold var $\left[\overline{g\left(w_{x}\right)} \mid \tau\right]$. In Appendix C (Theorem C.3), we show that the CV of $\widehat{Q(f, H)}$ for statistics with proportion $q$ is at most $(q(k-1))^{-0.5}$, which is essentially (within a single sample) our "gold standard" CV.

A ppswor sample with respect to $f(w)=w$ can be computed from a streamed (or distributed) aggregated data $\left\{\left(x, w_{x}\right)\right\}$, using a structure that only holds a sample-size number of keys [14, 36, 37]. To sample with respect to $f\left(w_{x}\right)$, we can simply replace the value $w_{x}$ of the element with key $x$ by $f\left(w_{x}\right)$. We can also compute a ppswor sample for sum statistics (when $f(w)=w$ ) over unaggregated $[10,12,18,23]$ distributed or streamed data, with similar efficiency (albeit with a caveat that when streaming we do not obtain exact frequencies $w_{x}$ for keys in our sample). Such efficient sampling over unaggregated data, however, is not possible for all $f$. For example, there are polynomial lower bounds on the structure size needed by a streaming algorithm that approximates frequency moments $Q\left(x^{p}, \mathcal{X}\right)$ with $p>2[1]$.

\section{SAMPLING FRAMEWORK}

We present a framework for sampling unaggregated datasets that extends ppswor sampling. Our sampling schemes can be realized in streamed or distributed settings by structures that only contain a cache $S$ or (current) sampled keys. In particular, the structure size never exceeds the final sample size.

A sampling scheme in our framework is specified by a mapping function of data elements to numeric scores. The mapping is specified by a distribution hDist, a random hash distribution $H$ so that with $h \sim H, h(x) \sim$ hDist and $h(x)$ are independent for different strings $x^{2}$ and a function Score $(g, r) \geq 0$ where $g$ is in the support of hDist, and $r>0$. We require that Score is monotone non-decreasing in $r$. We also require that for any $\tau>0$, for all $g$, there is small-enough $r$ such that $\operatorname{Pr}_{g \sim h \text { Dist }}[\operatorname{Score}(g, r)<\tau]>0$.

We use the Score function, together with $h \sim H$, to randomly map all data elements to numeric values as follows:

$$
\operatorname{ElementScore}(e):=\operatorname{Score}(h(e . k e y), \operatorname{Exp}[\text { e.val }]),
$$

where $\operatorname{Exp}[$ e.val $]$ is an independent exponential random variable with parameter e.val.

We define the seed of a key $x$

$$
\operatorname{seed}(x)=\min _{e \mid e . k e y=x} \operatorname{ElementScore}(e)
$$

to be the random variable that is the minimum score of all data elements with key $x$.

As with ppswor, we can obtain a fixed-threshold sample $S=\{x \mid \operatorname{seed}(x)<\tau\}$, which for a given $\tau$ includes all keys with seed $(x)<\tau$, or a fixed-size bottom- $k$ sample, which for a specified sample size $k$ includes the $k$ keys with smallest seed values and also the $(k+1)$ smallest seed value (for which we use the notation $\tau$ ).

We next express the distribution of $\operatorname{seed}(x)$ in terms of the function Score and the distribution hDist and establish useful properties that follow from our assumptions.

\footnotetext{
${ }^{2}$ Assumption made to streamline analysis. More limited independence suffices for many of our results. We note that in practice, hash representation can be considered part of the code and not part of the sketch and typically performance with non-perfect hash functions matches analysis.
} 
LEMMA 3.1.

(1) $\operatorname{seed}(x) \sim \operatorname{SeedDist}\left[w_{x}\right]$ where

$$
\text { SeedDist }\left[w_{x}\right]:=\operatorname{Score}\left(h \operatorname{Dist}, \operatorname{Exp}\left[w_{x}\right]\right) \text {. }
$$

(2) The random variables seed $(x)$ for $x \in \mathcal{X}$ are independent.

(3) For all $w>0$ and $\tau>0, \operatorname{Pr}_{r \sim \text { SeedDist }\left[w_{x}\right]}[r<\tau]>0$.

Proof. (1) We first consider seed $(x) \mid h$, which is the distribution of $\operatorname{seed}(x)$ conditioned on $h$. From monotonicity of Score and the property that the minimum of independent random variables Exp[e.val] used for scoring the elements with key $x$ is exponentially distributed with the sum of the values, we obtain that seed $(x) \mid h \sim \operatorname{Score}\left(h(x), \operatorname{Exp}\left[w_{x}\right]\right)$. The (unconditioned) distribution of $\operatorname{seed}(x)$ is specified by drawing $h \sim H$ and then drawing Score $\left(h(x), \operatorname{Exp}\left[w_{x}\right]\right)$. From our assumptions, $h(x) \sim$ hDist.

(2) The independence of seed $(x)$ for different keys follows from the properties of our ideal random hash functions $h \in H$.

(3) The positive probability of small seed $(x)$ follows from our assumptions on Score combined with the exponential distribution having positive CDF for any positive value.

The lemma implies that the joint distribution of $\operatorname{seed}(x)$ for $x \in \mathcal{X}$ depends only on the aggregated view $\left\{\left(x, w_{x}\right)\right\}$ and not on the way data elements are arranged or on the order in which they are processed.

COROLlary 3.2. Both with fixed-threshold or with fixed-size sampling, any key $x$ with $w_{x}>0$ has a positive probability to be included in the sample. In particular, any such key has a positive probability to have the minimum seed $(x)$.

\subsection{Estimation Overview}

Once we have the sample, we would like to apply estimators to it to approximate statistics $Q(f, H)$. As with ppswor, we use estimators that can be expressed as a sum over keys $x \in H$ of per-key estimates $\overline{f\left(w_{x}\right)} \geq 0$ of $f\left(w_{x}\right)$. Our estimates $\overline{f\left(w_{x}\right)}$ are 0 when the key $x$ is not sampled, and therefore it suffices to sum the estimates over sampled keys. For unbiasedness, it is necessary that any key with $f\left(w_{x}\right)>0$ is sampled with positive probability-we only consider $f$ such that $f(w)>0 \Rightarrow w>0$.

For estimation, we need information on the frequencies of sampled keys. Exact frequencies $w_{x}$ of sampled keys $x \in S$ can be computed in a second pass over the data. This allows us to compute the inclusion probability $\operatorname{Pr}[\operatorname{seed}(x)<\tau]$ and then use the inverse probability estimate $\overline{f\left(w_{x}\right)}=f\left(w_{x}\right) / \operatorname{Pr}[\operatorname{seed}(x)<\tau]$ as in (2). In a pure streaming (single sequential pass) setting, we will generally settle for a partial information on the frequency of a sampled key $x$ in the form of a random variable $c_{x} \leq w_{x}$. We will use $c_{x}$ with distribution parametrized only by $\tau$ and $w_{x}$ so that it is independent for different sampled keys. In particular, our fixed-size streaming schemes are designed so that the distribution of $c_{x}$ (conditioned on $\tau$ ) is the same as the distribution with fixed-threshold sampling with $\tau$. To derive estimators, we express sampling as a transform (which depends on $\tau$ ) from the distribution $w_{x}$ to the expected outcome distribution $c_{x}$. Our unbiased estimators $\overline{f\left(w_{x}\right)}$ are obtained by inverting this transform and expressed as function of $\tau$ and $c_{x}$. Because $c_{x}$ are independent for different keys, we have that conditioned on $\tau$ and a subset $Y \subset S$ of keys being included in the sample, the estimates $\overline{f\left(w_{x}\right)}$ for $x \in Y$ are independent. The transforms we obtain have a unique inverse, which means that our estimators are the optimal (minimum variance) unbiased and nonnegative sum estimators. Because the two-pass estimators (2) are also optimal, and rely on more information, the exact value $w_{x}$ instead of a sample from a distribution 
with parameter $w_{x}$, the variance of the streaming estimators is always at least that of the two-pass estimator.

In Appendix A, we establish, for our fixed-size (bottom- $k$ ) schemes, unbiasedness and nonpositive covariances of our per-key estimates under the above conditions. This allows us to express the variance of our estimators of statistics in terms of the distribution of $\tau$ and the per-key variance $\overline{f\left(w_{x}\right)}$ when conditioned on $\tau$. This allows us to extend the variance analysis of bottom- $k$ ppswor (Appendix C) to our unaggregated data schemes.

For estimation quality that approaches the "gold standard" (CV upper bound of $\left.(q k)^{-0.5}\right)$, we would need to sample each key $x$ with probability roughly proportional to $f\left(w_{x}\right)$. That is, to have element scoring function that roughly distributes like $1-\exp (-f(w) \tau)$. Our challenge is to identify when and how we can achieve this on streamed or distributed data using a small size structure.

\section{TWO-PASS SAMPLING ALGORITHMS}

We present our two-pass sampling scheme. The first pass identifies the set of sampled keys $S$. For fixed-threshold sampling, our sample contains all keys with scores below a specified threshold $\tau$. With fixed-size sampling, the sample contains the keys with $k$ smallest minimum element scores, for a specified sample size $k$. The operations on the summary structure that are needed to support a streamed or distributed summarization are as follows: Initializing the structure, processing a data element (and updating structure), and merging/composing summary structures of two datasets to obtain a summary structure for their union. Algorithm 1 specifies these operations for the first pass with fixed- $k$ sampling: The initialization is an empty sample with threshold value $\tau$ that upper bounds the scoring range. The element processing of $e$ first scores the element. It then tests if $e . k e y$ is in the current sample and if so updates its seed to the minimum of the current seed and the new element score. If e.key is not in the sample and the element score is below $\tau$, then e.key is placed in the sample with seed value equal to its current element score. If, as a result, the sample now includes $k+1$ keys, then the key with the largest seed is deleted and $\tau$ is updated to be its seed value. The merge procedure places all keys in the union in a new sample. If a key appeared in both samples, then we use the smallest of the seed values. Otherwise, we take the seed from the sample that includes the key. We also record the smallest of the $\tau$ values. If as a result we obtain a sample with more than $k$ keys, then we remove all but the $k$ keys with the smallest seed and set $\tau$ to be the $(k+1)$ st largest seed value. It is easy to verify that the sample at any point satisfies our definitions.

The respective operations for fixed- $\tau$ sampling structures are not shown and are simpler: The initialization is to an empty sample. The element processing places a key in the sample on the first element with score below $\tau$. Keys are never deleted from the sample. To merge two samples we take the union of their keys, and associate with each key that appeared in both samples the smaller of the seed values.

In the second pass, we compute the exact frequencies $w_{x}$ for each $x \in S$. Algorithm 2 specifies the structure and operations for streamed/distributed computation (for both fixed-size and fixedthreshold sampling).

\section{STREAM SAMPLING ALGORITHMS: DISCRETE DESIGN}

Our one-pass (streaming) schemes are designed for sequential processing of data elements. They also apply to multiple sharded streams as long as all elements with the same key are processed at the same shard. With stream sampling, to facilitate estimation, we need to collect partial information on the frequencies of sampled keys. Our discrete and continuous designs do this differently. In this section, we present the algorithms for the discrete design, which only applies when 

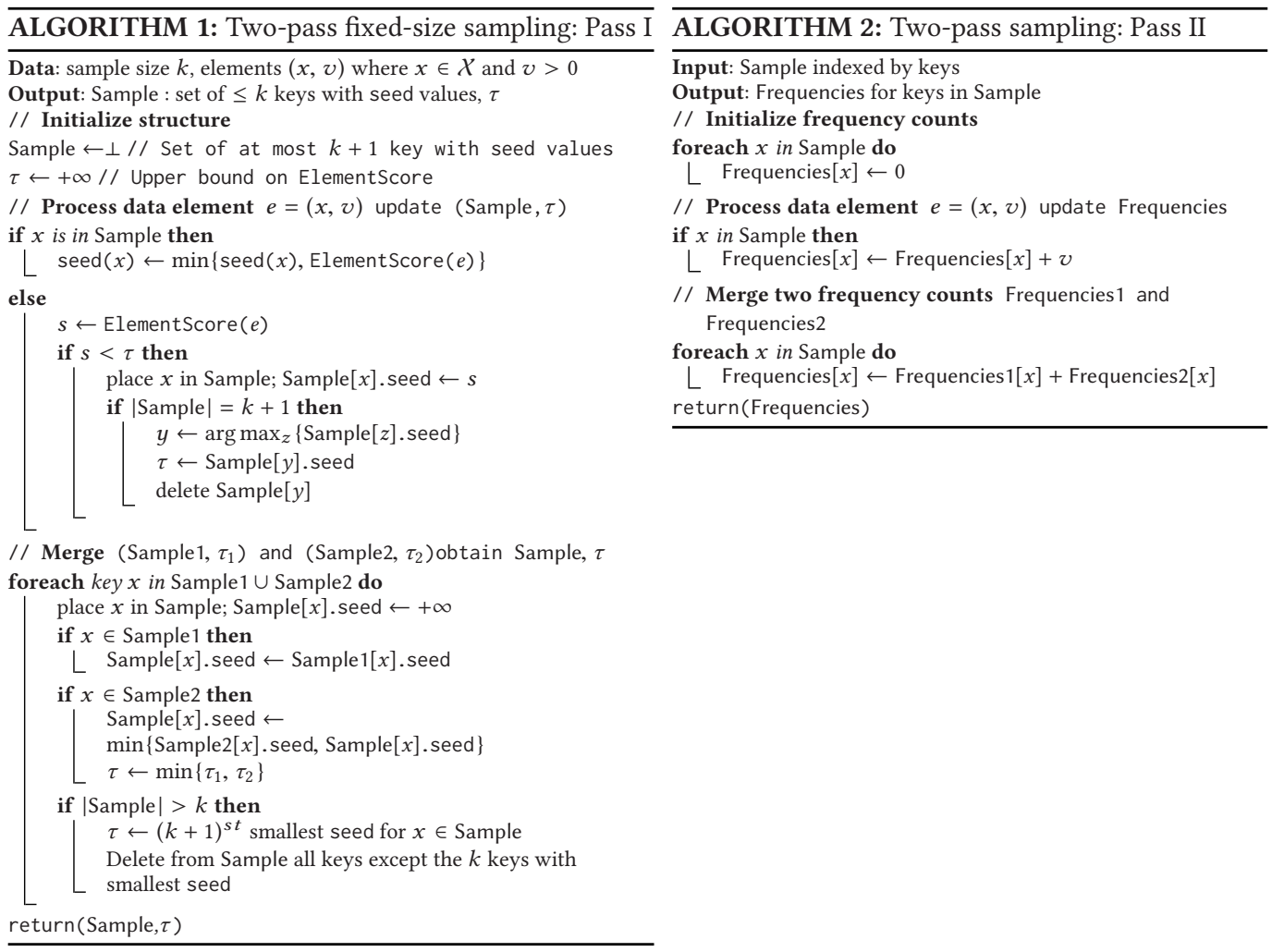

elements have uniform values (are unweighted). Our more general continuous design is presented in Section 8.

\subsection{Fixed-Threshold Stream Sampling}

The fixed-threshold stream sampling algorithm (discrete design) (Algorithm 3) maintains a set of initially empty counters that are indexed by "sampled" keys $S$. We process an element $e$ with key $x$ as follows. If $x \in S$, then $c_{x} \leftarrow c_{x}+1$. Otherwise, if ElementScore $(e)<\tau$, then $x$ is inserted to $S$ and $c_{x} \leftarrow 1$ is initialized. The computation is $O(1)$ per stream element.

\subsection{Fixed-Size Stream Sampling}

Algorithm 4 is a stream sampling algorithm with a fixed sample size $k$ (discrete design). The algorithm maintain a set counters (Counters) indexed by an (initially empty) set $S$ of sampled keys. We denote by $c_{x}$ (Counters $[x]$ ) the current count for $x \in S$. For each $x \in S$, we also maintain a lazily computed seed value seed $(x)$. Recall that we defined the seed value to be the minimum score of the elements we processed but note that our algorithm here maintains it in a lazy fashion by delaying the randomized scoring of element of keys that are currently in $S$.

To process an element $e$ with key $x$, we first check if $x \in S$ and if so, we increment $c_{x}$. Otherwise (if $x \notin S$ ), we score the element $y \leftarrow \operatorname{ElementScore}(e)$ and if the score is smaller than $\tau$, we insert $x \in S$ with $c_{x} \leftarrow 1$ and set the seed value seed $(x)$ to the score. As a result, we may have $|S|=k+1$ sampled keys. In this case, we would like to evict from $S$ the key with maximum seed. But the seeds are not fully evaluated yet, in that the current $\operatorname{seed}(x)$ only reflect the seed up to the first element that is currently counted in $c_{x}$. 

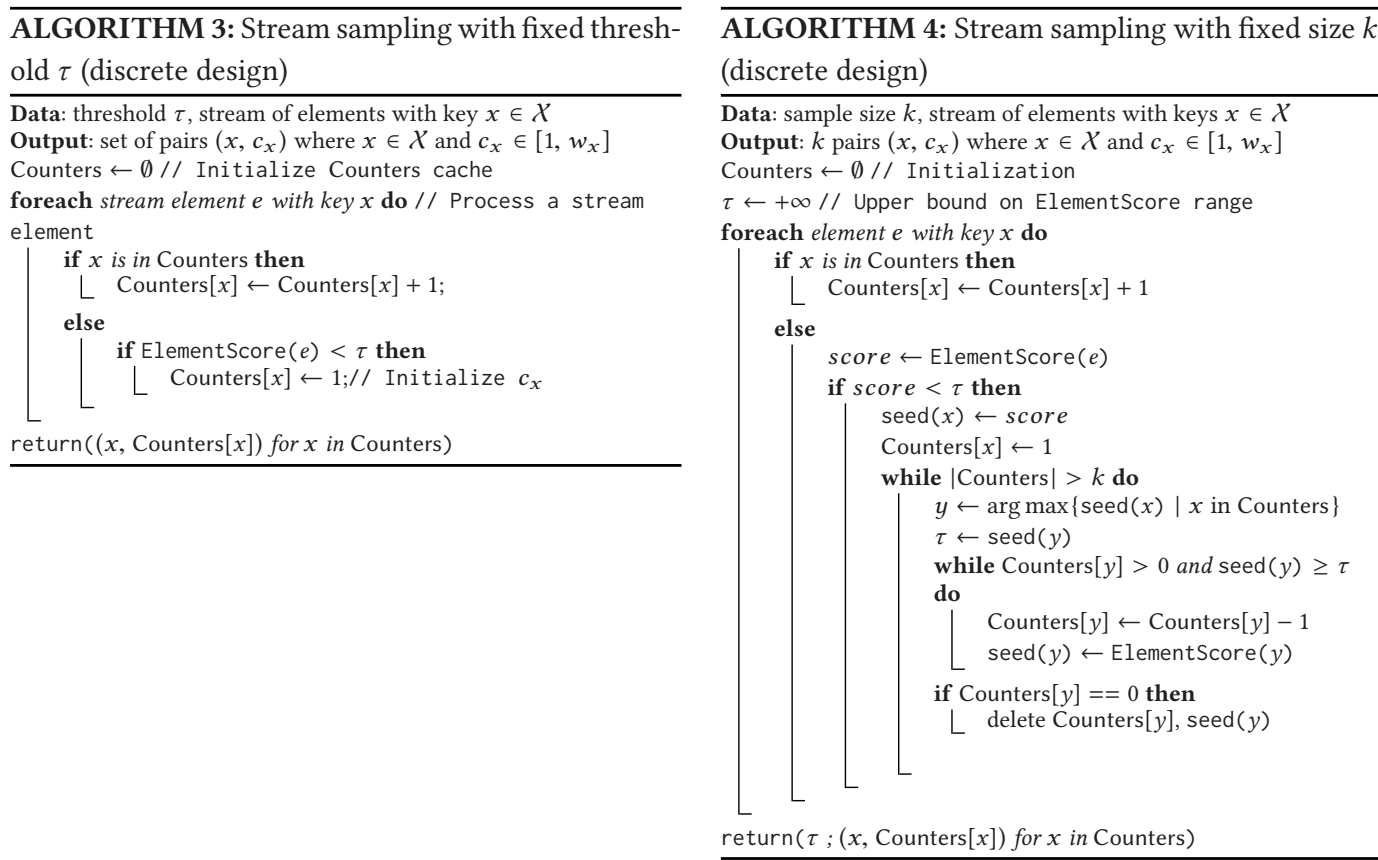

We repeat the following until a key is evicted. We pop from $S$ the key $y$ with maximum current seed and set $\tau \leftarrow \operatorname{seed}(y)$. The key $y$ is our current candidate for eviction, but we first need to score its unscored elements and see that it does not get a better (lower) seed value. To do so, we iterate decreasing the count $c_{y}$ and scoring "uncounted" elements until either the count becomes $c_{y}=0$ and $y$ is evicted or we obtain a score that is below $\tau$. In the latter case, we reinsert $y$ to $S$ with $\operatorname{seed}(y)$ equal to that score. We then look for a new candidate for eviction.

The algorithm may require more computation for some elements. Note however that each decrease of a counter can be "charged" to the processing of the element. So the amortized per-element work of Algorithm 4 is $O(1)$ plus the cost of priority queue operations of $O(\log k)$ (when a priority queue is used to hold keys by current seed value).

\section{DISTINCT AND SUM SAMPLING}

We cast two existing classic sampling schemes in our framework: Distinct, which is designed for cap $_{1}$ statistics and $\mathrm{SH}$, which is designed for $\operatorname{cap}_{\infty}$ (sum) statistics. Both our discrete-design and continuous-design element scoring functions generalize these two special cases.

\subsection{Distinct sampling}

A distinct sample is a uniform sample of active keys (those with $w_{x}>0$ ), meaning that conditioned on sample size $k$, all subsets of active keys are equally likely. We use $H$ such that hDist $:=U[0,1]$ and specify $\operatorname{Score}(g, r)=g$. With $h \sim H$, the score of an element $e$ with key $x$ is ElementScore $(e)=h(x)$. Since all elements of the same key $x$ have the same score, $\operatorname{seed}(x)=$ $h(x)$ with distribution SeedDist $\sim U[0,1]$.

When we sample with respect to a fixed threshold $\tau$, we retain all active keys with $h(x)<$ $\tau$. When using a fixed sample size $k$, the scheme is the following (distinct) variant of reservoir sampling [31]: For each stream element with key $x$ we compute $h(x)$ and retain the $k$ keys with smallest hash values. 
With stream distinct sampling (Algorithms 3 or 4), we obtain $c_{x}$ that is equal to the exact frequency $w_{x}$ for each sampled key $x$. This is because any key that enters our cache does so on the first element of the key. If a key is evicted, (in the fixed $k$ scheme), then it can never re-enter. We also have that for all keys with $w_{x}>0$, the probability that $x$ is sampled is $\operatorname{Pr}[\operatorname{seed}(x)<\tau]=\tau$. We can therefore apply the inverse probability estimator (2):

$$
\widehat{Q(f, H)}=\frac{1}{\tau} \sum_{x \in S \cap H} f\left(w_{x}\right) .
$$

With distributed sampling (Algorithm 1) and the particular case of distinct $\left(\operatorname{cap}_{1}\right)$ statistics $(f(w)=$ 1 when $w>0$ ) we obtain the values $f\left(w_{x}\right)=1$ for all active keys from the first pass and we can use the estimator (5) without the need for a second pass.

In terms of estimate quality, distinct sampling is optimized for distinct statistics. In particular, $Q\left(\overline{\operatorname{cap}_{1}, \mathcal{X}}\right)$ has $\mathrm{CV}$ upper bounded by $(k-1)^{-0.5}[5,6]$ and for a segment $H$ with proportion $q$ of distinct keys, $\left.Q \overline{\left(\operatorname{cap}_{1}, H\right.}\right)$ has $C V$ upper bounded by $(q(k-1))^{-0.5}$, as it is equivalent to the ppswor estimator.

For general $\operatorname{cap}_{T}$ statistics, however, the CV grows rapidly with $T$ (we shall see it is $\propto \sqrt{T}$ ). This is because our uniform sample of active keys can easily miss keys with high $f\left(w_{x}\right)$ values that contribute more to the statistics.

\subsection{Sample and Hold (SH)}

Classic SH, with fixed sampling threshold $\tau$ or with fixed sample size $k[18,23]$, is specified for uniform element values, so that $w_{x}$ is the number of elements with key $x$. Casted in our framework, we use $\operatorname{Score}(g, r):=r$ (The distribution hDist is not used) and we get ElementScore $(e) \sim \operatorname{Exp}[1]$. Note that each key $x$ has $w_{x}$ independent scores drawn and the minimum, $\operatorname{seed}(x) \sim \operatorname{Exp}\left[w_{x}\right]$ is exponentially distributed with parameter $w_{x} .(\operatorname{SeedDist}[w]:=\operatorname{Exp}[w])$. Therefore, as observed in Reference [12], the SH sample is actually a ppswor sample with respect to the frequencies $w_{x}$ [36, 37].

The two-pass distributed algorithm (Algorithms 1 and 2) provide us with exact frequencies $w_{x}$, and we can apply the ppswor estimator (2).

With stream sampling (Algorithm 3 and Algorithm 4), the final count of a key $x$ has $c_{x} \leq w_{x}$, where $w_{x}-c_{x}+1$ is geometric with parameter $1-\exp (-\tau)$, truncated at $w_{x}+1$ (probability of $c_{x}=0$ is $\left.\exp \left(-\tau w_{x}\right)\right)$. An unbiased estimator for statistics $Q(f, H)$ from an SH sample is $[12]^{3,4}$ :

$$
\widehat{Q(f, H)}=\frac{1}{1-\exp (-\tau)} \sum_{x \in S \cap H}\left(f\left(c_{x}\right)-f\left(c_{x}-1\right) \exp (-\tau)\right) .
$$

Note that this estimator is nonnegative when $f$ is monotone non-decreasing. ${ }^{5}$ This is because for all $i>0, f(i)-f(i-1) \exp (-\tau)>0$.

For cap statistics with small $T$, a ppswor sample can have CV that far exceeds our $(q k)^{-0.5}$ target: When the frequency distribution is highly skewed, the ppswor sample would be dominated by heavy keys. This means that segments with a large proportion of the $\operatorname{cap}_{T}$ statistics that mostly include keys with low frequencies would have a disproportionally small representation in the sample and thus large errors.

\footnotetext{
${ }^{3}$ Estimators for a related scheme (where elements are drawn with replacement) were presented in Reference [1].

${ }^{4}$ The estimators in Reference [12] were presented for $U[0,1]$ instead of Exp[1] element scores. But the two are equivalent due to monotone mapping that preserves the minimum.

${ }^{5}$ With fixed-size sampling, we can instead use here the stratified value $1-\exp (-\tau)=k /\left(k+\sum_{x \in X} w_{X}-\sum_{x \in S \cap X} c_{X}\right)$.
} 


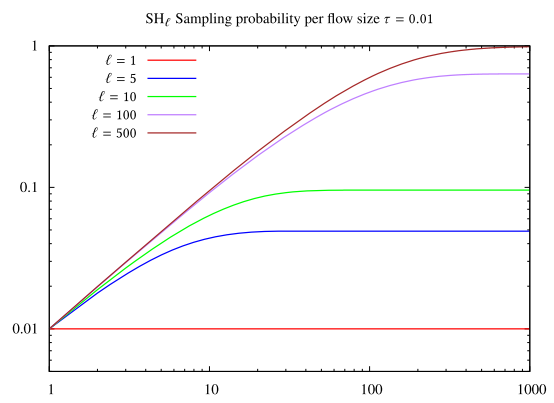

Fig. 1. $\mathrm{d}$-SH $\mathrm{SH}_{\ell}$ sampling probability ( $y$-axis) per key frequency $w$ ( $x$-axis), for selected values of $\ell(\tau=0.01)$. Note that for $w \gg \ell \log \ell$ probability is constant and for $w \ll \ell$, probability is proportional to $w$. We can see that the probability is close to being proportional to $\min \{w, \ell\}$, which is what we want for estimating cap $\ell$ statistics.

\section{DISCRETE DESIGN FOR CAP FUNCTIONS}

Our discrete schemes are applied to data elements with uniform values. The schemes are parametrized by an integer $\ell \geq 1$. Distinct sampling is d-SH and classic $\mathrm{SH}$ is $\mathrm{d}-\mathrm{SH}_{\infty}$. In general, $\mathrm{d}-\mathrm{SH}_{\ell}$ is designed to estimate well frequency cap statistics with $T \approx \ell$.

The d-SH $\mathrm{SH}_{\ell}$ element scoring function for an element $e$ with key $x$ draws a uniform random bucket $b \sim U[1, \ldots, \ell]$ and returns a hash of the pair $\operatorname{Hash}(x, b) \sim U[0,1]$. Note that the buckets are independent for different elements with key $x$,

$$
\text { ElementScore }(e) \leftarrow \operatorname{Hash}(\lfloor(\ell * \operatorname{rand}())\rfloor, x) \text {. }
$$

We define $\operatorname{seed}(x)$ (3) to be the minimum score of an element with key $x$. When $\ell=1$, the seed distribution is uniform for all keys with $w_{x}>0$. More generally, we can see that the element scoring (7) provides $w_{x}$ attempts to hit $\ell$ buckets for each key to obtain a lower seed. That way, keys with more elements are more likely to have a lower seed and be sampled but with diminishing return: Keys where $w_{x} \ll \min \left\{\ell, \tau^{-1}\right\}$ are sampled with probability roughly proportional to $w_{x}$ whereas keys with $w_{x} \gg \min \left\{\ell, \tau^{-1}\right\}$ have a roughly constant inclusion probability regardless of frequency. Also note that when the cap parameter is large relative to the inverse sampling threshold $\ell \gg \tau^{-1}$, $\mathrm{d}-\mathrm{SH}_{\ell}$ is similar to $\mathrm{d}-\mathrm{SH}_{\infty}$. Figure 1 illustrates these properties by showing the sampling probability of a key as a function of $w_{x}$, for selected values of the parameter $\ell$.

\subsection{Estimators for $\mathrm{d}-\mathrm{SH}_{\ell}$}

The output of our stream sampling algorithm is a threshold value $\tau$ and a set $S$ of pairs of the form $\left(y, c_{y}\right)$, where $y \in X$ and $c_{y} \in\left[1, w_{y}\right]$.

Coefficient form. We express our estimators as vectors $\boldsymbol{\beta}^{(f, \tau, \ell)}$, which depends on $f$, the threshold $\tau$, and the parameter $\ell$. The $i$ th entry $\boldsymbol{\beta}_{i}^{(f, \tau, \ell)}$ is the contribution to the estimate of a key with count $i$. The estimate on the statistics $Q(f, H)$ is then

$$
\widehat{Q(f, H)}=\sum_{x \in S \cap H} \beta_{c_{x}} .
$$

The distinct sample $\left(\ell=1\right.$ ) estimator (5) is expressed using $\beta_{i} \equiv f_{i} \tau^{-1}$ (using the notation $f_{i} \equiv$ $f(i))$ whereas the classic SH estimator $(\ell=+\infty)(6)$ is expressed using $\beta_{i} \equiv \tau^{-1}\left(f_{i}-f_{i-1}(1-\tau)\right)$. We seek estimators of this form for general $\ell$ that are unbiased, admissible, and nonnegative $\beta \geq 0$ when $f$ is non-decreasing. 
Probability vector $\phi$. Let $\phi_{i}$ be the probability that the $i$ th element of the same key was the first one to get counted by $\mathrm{d}-\mathrm{SH}_{\ell}$. The vector $\phi$ depends on the parameters $\ell$ and $\tau$.

For $\ell=1$, we have the closed form $\phi_{1} \equiv \tau$ and $\phi_{i}=0$ for $i>1$. For $\ell=+\infty$, we have $\phi_{i}=(1-$ $\tau)^{i-1} \tau$.

To express $\phi$ for general $\ell$, we let $a_{i j}$ be the probability that we used exactly $j \leq \min \{\ell, i\}$ buckets in the first $i$ elements with a certain key.

By definition $a_{0 i} \equiv 0$ when $i \geq 1, a_{i j} \equiv 0$ when $j>\min \{\ell, i\}$, and $a_{1,0}=0$. Otherwise, $a_{1,1}=1$ and for $i>1, j \leq \min \{\ell, i\}$, the values can be computed from the relation

$$
a_{i j}=a_{i-1, j} \frac{j}{\ell}+a_{i-1, j-1} \frac{\ell-j+1}{\ell} .
$$

Note that as $i$ grows, the vectors $a_{i}$. converge to a vector that has all entries 0 except $a_{i \ell}=1$. It therefore suffices to compute these entries only until $i=O(\ell \log (\ell))$ (this is a "coupon collector" problem-with high probability all buckets are hit by then). For larger values of $i$ we can use the vector $a_{i} .=(0, \ldots, 0,1)$.

We can now write

$$
\phi_{i}=\tau \sum_{j=1}^{\min \{i-1, \ell-1\}} a_{i-1, j}(1-\tau)^{j} \frac{\ell-j}{\ell} .
$$

Note that it always suffices to compute only the

$$
M=O\left(\min \left\{\ell \log \ell, \tau^{-1} \log \tau^{-1}\right\}\right)
$$

first entries of $\phi$, as the tail of $\phi$ vanishes beyond that.

A two-pass estimator. The probability that a key $x$ is sampled (illustrated in Figure 1) is

$$
\Phi_{\tau, \ell}\left(w_{x}\right) \equiv \sum_{j=1}^{w_{x}} \phi_{j} .
$$

If we use a two-pass scheme (Section 4), then we can apply the inverse probability estimator (2) $\widehat{Q(f, H)}=\sum_{x \in S \cap H} \frac{f\left(w_{x}\right)}{\Phi\left(w_{x}\right)}$.

Inverting the sample counts. We now derive a streaming estimator. We use the notation $o_{i}=$ $\left|\left\{x \in S \cap H \mid c_{x}=i\right\}\right|$ (the "observed" count) for the random variable that is the number of keys $x \in S \cap H$ with $c_{x}=i$. Let $m_{i}=\left|\left\{x \in H \mid w_{x}=i\right\}\right|$ be the number of keys in $H$ with count $w_{x}=i$. Our statistics (1) can be expressed as $Q(f, H)=f^{T} \boldsymbol{m}$. We have the relation $\mathrm{E}\left[o_{i}\right]=\sum_{j \geq i} \phi_{j-i+1} m_{j}$ and can write

$$
\mathrm{E}[\boldsymbol{o}]=Y^{(\boldsymbol{\phi})} \boldsymbol{m} .
$$

We use the notation $Y^{(\boldsymbol{v})}$ for an upper triangular matrix that corresponds to a vector $\boldsymbol{v}$, such that $\forall, j \geq i,\left[Y^{(\boldsymbol{v})}\right]_{i j} \equiv v_{j-i+1}$.

We have $\boldsymbol{m}=\left(Y^{(\boldsymbol{\phi})}\right)^{-1} \mathrm{E}[\boldsymbol{o}]$. Therefore, from linearity, $\hat{\boldsymbol{m}} \equiv\left(Y^{(\boldsymbol{\phi})}\right)^{-1} \boldsymbol{o}$ is an unbiased estimator of $\boldsymbol{m}$. Therefore, to compute the estimate we need to invert $Y^{(\phi)}$.

The inverse of the matrix $Y^{(\phi)}$ has the same upper triangular structure, and can be expressed as $Y^{(\psi)}$ with respect to another vector $\psi$. To compute $\psi$, we consider the constraints $Y^{(\psi)} Y^{(\phi)}=I$ obtained from the product of the first row of $Y^{(\psi)}$ with the columns of $Y^{(\phi)}$. We obtain the equations $\psi_{1}=\phi_{1}^{-1}$, and for $i>1$,

$$
\sum_{j=1}^{i} \psi_{j} \phi_{1+i-j}=0
$$


This allows us to iteratively solve for $\psi_{i}$ after computing $\psi_{j}$ for $j<i$ using

$$
\psi_{i}=\phi_{1}^{-1}\left(-\sum_{j=1}^{i-1} \phi_{1+i-j} \psi_{j}\right) .
$$

For distinct sampling we have $\psi_{1}=\tau^{-1}$ and $\psi_{i}=0$ for $i>1$. For classic SH [12] we have $\psi_{1}=\tau^{-1}$, $\psi_{2}=-(1-\tau) \tau^{-1}$, and $\psi_{i}=0$ for $i \geq 2$. In general, however, $\psi$ can have many non-zero entries.

We show the following:

THEOREM 7.1. The estimator $\overline{Q(f, H)}=\sum_{x \in S \cap H} \beta_{c_{x}}$, where

$$
\beta_{i}^{(f, \tau, \ell)} \equiv \sum_{j=1}^{i} \psi_{j} f_{i-j+1}
$$

is unbiased.

Proof. By substituting $\hat{\boldsymbol{m}}=Y^{(\psi)} \boldsymbol{o}$ in $Q(f)=f^{T} \boldsymbol{m}$, we obtain the estimator $\widehat{Q(f)}=f^{T} Y^{(\psi)} \boldsymbol{o}$.

The unbiased estimate for $m_{i}$ is

$$
\hat{m}_{i}=\sum_{j \geq i} o_{j} \psi_{j-i+1}
$$

The unbiased estimate for the contribution of keys with $i$ elements to the statistics is

$$
f_{i} \hat{m}_{i}=f_{i} \sum_{j \geq i} o_{j} \psi_{j-i+1} \text {. }
$$

Therefore, the total contribution, and expressed in terms of $o_{i}$ is

$$
\sum_{i} \sum_{j \geq i} f_{i} o_{j} \psi_{j-i+1}=\sum_{i} o_{i} \sum_{j=1}^{i} \psi_{j} f_{i-j+1} .
$$

Since the inverse is unique, our estimator is the only unbiased estimator of this form and thus also admissible (minimum variance of this form). Note that when we only compute the first $M$ entries of $\psi$, we limit the sum expression to range from 1 to $\min \{M, i\}$. In applications, the coefficients $\beta$ only need to be computed for $i$ such that there is at least one key $x$ in the sketch with $c_{x}=i$.

We show that the estimates are nonnegative when $f$ is monotone non-decreasing:

THEOREM 7.2. When $f$ is monotone non-decreasing, then for all $\ell$ and $\tau, \boldsymbol{\beta}^{(f, \tau, \ell)} \geq 0$.

Proof. We first claim that any prefix sum of $\psi$ is positive. That is,

$$
\forall j \geq 1, \sum_{i=1}^{j} \psi_{i}>0 .
$$

We prove the claim by induction on $i$. The base case of the induction has $\psi_{1}=\phi_{1}^{-1} \equiv \tau^{-1}>0$. We now show that $\sum_{i=1}^{h} \psi_{i}>0$ if the claim (10) holds for all $j<h$. We have

$$
\begin{aligned}
0 & =\sum_{j=1}^{h} \psi_{j} \phi_{h-j+1} \\
& =\phi_{1} \sum_{i=1}^{h} \psi_{j}+\sum_{j=1}^{h-1}\left(\sum_{i=1}^{j} \psi_{i}\right)\left(\phi_{h-j+1}-\phi_{h-j}\right) .
\end{aligned}
$$


Rearranging, we obtain

$$
\phi_{1} \sum_{i=1}^{h} \psi_{j}=\sum_{j=1}^{h-1}\left(\sum_{i=1}^{j} \psi_{i}\right)\left(\phi_{h-j}-\phi_{h-j+1}\right) .
$$

We now argue that the right-hand side is nonnegative. In fact, each summand, and each term in the product are nonnegative. Nonnegativity of the sums $\sum_{i=1}^{j} \psi_{i}$ follows from the induction hypothesis for $j<h$. Nonnegativity of the differences $\phi_{h-j}-\phi_{h-j+1}$ for $j<h$ follows from $\phi_{i} \geq 0$ being non-increasing (recall that $\phi_{i}$ is the probability that the $i$ th element of a key is the first one to be counted). Now, the left-hand side is nonnegative and $\phi_{1}=\tau^{-1}>0$. Therefore, $\sum_{i=1}^{h} \psi_{j} \geq 0$.

We are now ready to establish the nonnegativity of the estimation coefficients:

$$
\begin{aligned}
\beta_{i} & =\sum_{j=1}^{i} \psi_{j} f_{i-j+1} \\
& =\sum_{h=1}^{i}\left(f_{h}-f_{h-1}\right) \sum_{j=1}^{i-h+1} \psi_{j} .
\end{aligned}
$$

The nonnegativity of the right-hand side follows from monotonicity of $f$ and our claim (10) on the nonnegativity of the $\psi$ prefix sums.

\section{CONTINUOUS DESIGN FOR CAP FUNCTIONS}

We now present our continuous $\mathrm{SH}_{\ell}$ sampling schemes, which generalizes the $\mathrm{SH}_{\infty}$ scheme in Reference [10]. Our continuous design offers the following advantages over the discrete design (Section 7) even when applied to uniform values. First, fixed sample-size sampling no longer requires explicitly maintain a lazy seed $(x)$ for cached keys as we did in Algorithm 4: The lazy value is implicitly captured by the current threshold $\tau$. Second, the estimator can be expressed in terms of $f$ and its derivative. Last, the continuous schemes facilitates multi-objective samples (Section 9).

The scheme with parameter $\ell$ is specified by a hash family $H$ with hDist $:=U[0,1 / \ell]$ and

$$
\text { Score }(g, r):=r \leq 1 / \ell ? g: r .
$$

The score function returns $g$ if $r \leq 1 / \ell$ and $r$ otherwise.

We use a hash function KeyBase $\sim H$ and score each element $e=(x, v)$ with key $x$ and a value $v>0$ using

$$
\operatorname{ElementScore}(e):=\operatorname{Score}(\operatorname{KeyBase}(x), \operatorname{Exp}[v]) \equiv(r \sim \operatorname{Exp}[v]) \leq 1 / \ell \text { ? KeyBase }(x): r \text {. }
$$

We next consider the distribution SeedDist $[w]$ of $\operatorname{seed}(x)$ for a key $x$ with $w_{x}=w$.

LEMMA 8.1.

$$
\text { SeedDist }[w]:=(r \sim \operatorname{Exp}[w]) \leq 1 / \ell ? U[0,1 / \ell]: r .
$$

Equivalently, we return $U[0,1 / \ell]$ with probability $\left(1-e^{-w / \ell}\right)$ and $1 / \ell+\operatorname{Exp}[w]$ otherwise.

Proof. From Lemma 3.1, SeedDist $[W]:=$ Score(hDist, Exp $[w])$. We use Equation (11) to obtain the first part of the claim.

If $r \sim \operatorname{Exp}[w]$ is such that $r<1 / \ell$, which happens with probability $1-e^{-w / \ell}$, then the seed is KeyBase $(x)$. Otherwise, seed $(x)=r$. We use the memoryless property of the exponential distribution, which implies that the conditional distribution of $r$ given that $r>1 / \ell$ is $1 / \ell+\operatorname{Exp}[w]$. So with probability $e^{-w / \ell}$, the distribution is $1 / \ell+\operatorname{Exp}[w]$.

Qualitatively, when $w_{x} \ll \ell$, the seed is close to exponentially distributed with parameter $w_{x}$, which is ppswor. When $w_{x} \gg \ell$, the seed is uniform, which results in distinct sampling. We obtain 


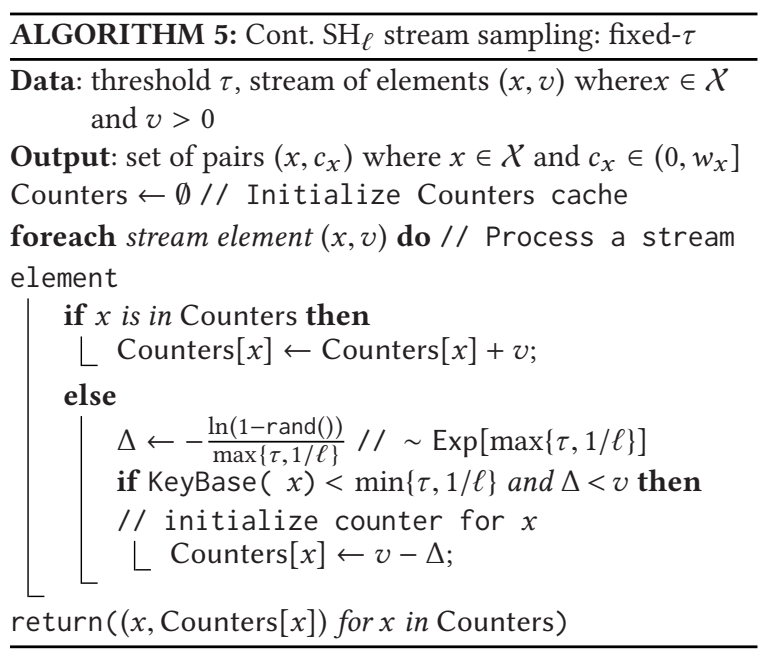

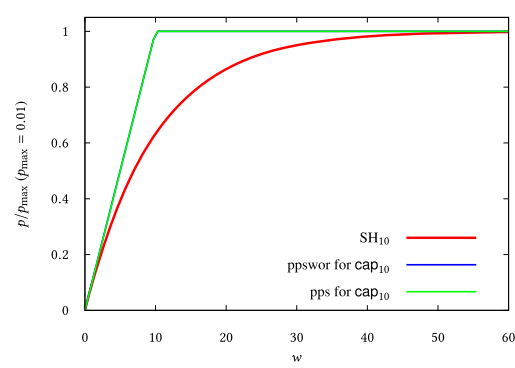

Fig. 2. (Relative) sampling probabilities as a function of the frequency $w_{x}$ for $\mathrm{SH}_{10}$, pps, and ppswor, computed with respect to $\operatorname{cap}_{10}$. The $y$ axis shows the inclusion probability as a fraction of the maximum inclusion probability. For all schemes we normalized the threshold to be such that the inclusion probability maximizes at 0.01 . The ppswor and the $\mathrm{SH}_{10}$ plots overlap.

the property that the sampling probability a key $x$ is roughly proportional to $\operatorname{cap}_{\ell}\left(w_{x}\right)$, which is needed to approach the "gold standard" CV.

\subsection{Estimation for Two-Pass Sampling}

With two-pass sampling (Section 4 ) we obtain the frequencies $w_{x}$ of sampled keys. We compute the probability, conditioned on $\tau$, that a key $x$ is sampled.

LEMMA 8.2. When sampling with element scoring function (12),

$$
\operatorname{Pr}[\operatorname{seed}(x)<\tau] \equiv \Phi_{\tau, \ell}\left(w_{x}\right)=\left(1-e^{-w_{x} \max \{1 / \ell, \tau\}}\right) \min \{1, \tau \ell\} .
$$

Proof. If $\tau \ell<1$, then $\operatorname{seed}(x)<\tau$ if $\operatorname{Exp}\left[w_{x}\right]<1 / \ell$ and then KeyBase $(x)<\tau$. These two events are independent and have joint probability $\left(1-e^{-w_{x} / \ell}\right) \tau \ell$. If $\tau \ell \geq 1$, then $\operatorname{seed}(x)<\tau$ if $\operatorname{Exp}\left[w_{x}\right]<\tau$, which has probability $\left(1-e^{-\tau w_{x}}\right)$. We express the combined probability to obtain the claim.

This allows us to apply the inverse probability estimator, as in Equation (2), to estimate a statistics

$$
\widehat{Q(f, H)}=\sum_{x \in S \cap H} \frac{f\left(w_{x}\right)}{\Phi_{\tau, \ell}\left(w_{x}\right)} .
$$

We show the following (Proof provided in Appendix E.1):

Theorem 8.3. The $C V$ of $Q \overline{\left(c a p_{T}, H\right)}$ (the estimator (14) applied to an $\mathrm{SH}_{\ell}$ sample with exact frequencies $\left.w_{x}(x \in S)\right)$ is at most

$$
C V\left[Q \overline{\left(\operatorname{cap}_{T}, H\right)}\right] \leq\left(\frac{e}{e-1} \frac{\max \{T / \ell, \ell / T\}}{q(k-1)}\right)^{0.5} .
$$

When $\ell=T$, we obtain a bound of at most 1.26 times the CV bound of $\left(\frac{\max \{T / \ell, \ell / T\}}{q(k-1)}\right)^{0.5}$ we can obtain for samples computed over the aggregated data (Section 2). When $\ell=\Theta(T)$, the CV is $O\left((q(k-1))^{-0.5}\right)$ and the upper bound degrades smoothly with the disparity $\max \{T / \ell, \ell / T\}$ between $\ell$ and $T$. Also note that the increased CV due to the constant $e /(e-1)$ and the disparity arise from a worst-case analysis and are not inherent. 
Figure 2 shows the relative inclusion probabilities as a function of the frequency $w_{x}$ for $\mathrm{SH}_{10}$, and pps and ppswor with respect to $\operatorname{cap}_{10}\left(w_{x}\right)$. The "gap" between the ratios for $\mathrm{SH}_{10}$ and for the pps/ppswor (which are realizable on aggregated data) illustrates our loss relative to the "gold standard" CV. We can see that the gap is larger for frequencies that are around the cap parameter of 10 , and maximizes at the ratio $(1-1 / e)$. In this sense, data with many keys with frequency close to the cap thresholds are the "worst case" for the variance.

Fixed-sample-size sampling is provided as Algorithm 6. To maintain a fixed-size sample, the threshold is decreased when there are $k+1$ cached keys, to the point needed to evict a key. The algorithm "simulates" the end result of working with the lower threshold to begin with.

The eviction step is as follows. We draw and fix some "randomization" and compute for each cached key the threshold needed to evict the key. The randomization for key $x$ is captured by $u_{x}$ and $r_{x}$. We then compute $z_{x}$, which is the maximum threshold value that is needed to evict $x$ with respect to that randomization. We then take the new threshold to be the maximum $z_{x}$ over keys. One key (the one with maximum $z_{x}$ ) is evicted. For remaining keys, $c_{x}$ (Counters $[x]$ ) is updated according to the same $u_{x}, r_{x}$.

We elaborate on how $z_{x}$ is determined when the current threshold is $\tau$. The key $x$ can be viewed as having a score (computed to the point the key entered the cache) that is at most $\tau$. We can consider the distribution of the score given that it is at most $\tau$ : With the randomization, we can take it as $u_{x} \tau$. A necessary requirement for $x$ to be evicted is that the new threshold $\tau^{*}$ is below $u_{x} \tau$, so we have $z_{x}<u_{x} \tau$. Conditioned on $\tau^{*}<u_{x} \tau$, we can treat this as processing an element with a new (uncached) key $x$ and frequency $c_{x}$. We consider the threshold value $\tau^{*}$ needed for the key to enter the cache. We simply reverse the entry rule: If $-\ln \left(1-r_{x}\right) / c_{x} \geq \ell^{-1}$, then the key would enter the cache when $\tau^{*} \geq-\ln \left(1-r_{x}\right) / c_{x}$. If $-\ln \left(1-r_{x}\right) / c_{x}<\ell^{-1}$, then the key would enter the cache if and only if $\tau^{*} \geq$ KeyBase $(x)$, with count $c_{x}-\ell\left(-\ln \left(1-r_{x}\right)\right)$.

\subsection{Streaming}

The streaming algorithms compute a sample $S$ of cached keys and "partial frequency" $c_{x} \leq w_{x}$ for each $x \in S$. We can view the element score as being continuously assigned to the mass $w_{x}$. The count $c_{x} \leq w_{x}$ is the frequency processed after the first point that the score got below $\tau$.

Algorithm 5 performs fixed-threshold stream sampling. When processing an element $e=(x, v)$ with a cached key, we update $c_{x} \leftarrow c_{x}+v$. Otherwise, we fix the "randomization," which allows us to obtain an element score as a decreasing function of a value of the element. We can then compute the minimum value $\Delta$ that is needed for the score to be below $\max \{1 / \ell, \tau\}$. If $v \leq \Delta$, then we break. Otherwise, if $\tau<1 / \ell$, then we break if KeyBase $(x) \geq \tau$. Finally, we initialize a counter $c_{x} \leftarrow v-\Delta$. Breaking is equivalent to the element score of $e=(x, v)$ being above $\tau$. If we do not break, then we insert the key into the cache with the remaining value of $v-\Delta$.

We now express the distribution of $c_{x}$ (Counters $[x]$ ) and verify that it satisfies our requirement (Section 3) that for any key $x$, it only depends on $w_{x}, \ell$, and $\tau$. Recall that for fixed-threshold $\mathrm{SH}_{\ell}$ we use the specified $\tau$, whereas with fixed-cache size $\mathrm{SH}_{\ell}$, the statement is conditioned on the randomization on all other keys, which determines $\tau$ when $x \in S$. The proof is provided in Appendix B.

Theorem 8.4. With fixed- $\tau$ SH $H_{\ell}$ (Algorithm 5) and fixed-k $S H_{\ell}$ (Algorithm 6), for any key $x, c_{x} \sim$ $\max \left\{0, w_{x}-\phi\right\}$, where $\phi$ has density

$$
\phi(y)=\tau \exp (-y \max \{1 / \ell, \tau\})
$$

in the interval $y \in\left[0, w_{x}\right]$. 


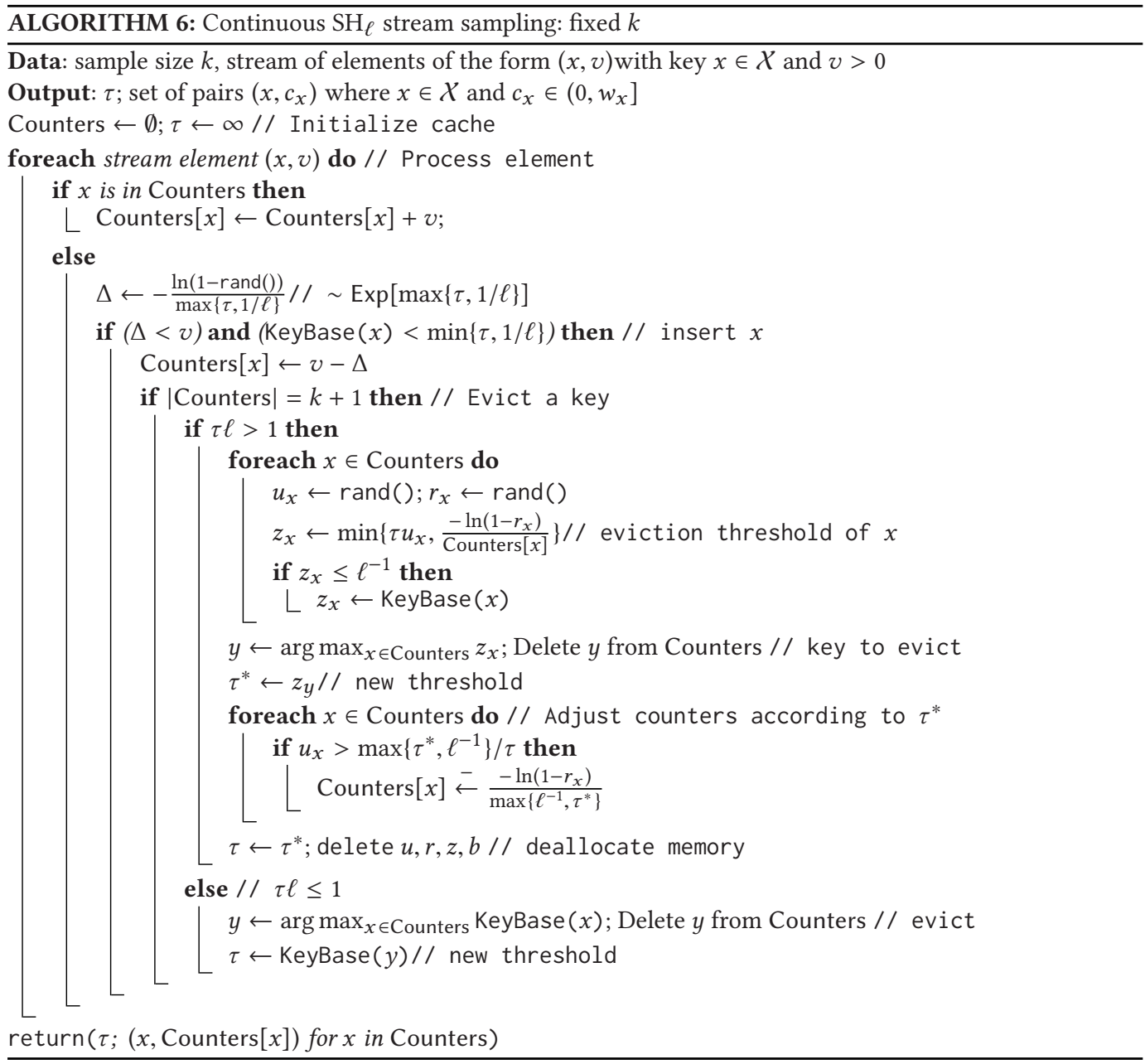

Batch Evictions Optimization. Each decrease of the threshold involves scanning all keys in the cache. The expected total number of evictions, however, is at most $k\left(\ln n+\ln \left(\sum_{e}\right.\right.$ e.val $/$ $\left.\min _{e} e . v a l\right)$ ), where $n$ is the number of distinct keys. When the threshold $\tau$ gets below $1 / \ell$, the sample contains the set of keys with smallest KeyBase $(x)$ values. The order on these values is a random permutation and after $i$ distinct keys are processed the probability that a new key updates the threshold is $\min \{1, k / i\}$. The sum over $i$ is at most $k \ln n$. When the threshold is above $\tau$ we can obtain score below $\tau$ after in expectation processing total frequency about $1 / \tau$. Initially, $\tau$ in expectation is at most $k / \min _{e}$ e.val The decrease in the threshold is in expectation a factor of $k /(k+1)$ (the other cached keys have exponentially distributed seed values conditioned on being below $\tau$ and we take the largest one).

To reduce amortized eviction cost, we can use a slight modification of the algorithm that evicts $\delta k$ keys when the cache is full (a fraction of the cache). The modification uses the $\delta$ th largest $z_{x}$ instead of the maximum one as the new $\tau^{*}$. All keys $y$ with $z_{y} \geq \tau$ are then evicted. The new threshold is $\tau^{*}$. The computation of the estimators, which we present next, is with respect to the current threshold and is the same with the batched evictions or one at a time eviction. 


\subsection{Estimators for Continuous $\mathrm{SH}_{\ell}$}

We seek an unbiased and nonnegative estimator in a coefficient form, that is, a function $\beta^{(f, \tau, \ell)}(c)$ defined for any $c>0$ and we use the estimator

$$
\overline{Q(f, H)}=\sum_{x \in H \cap S} \beta^{(f, \tau, \ell)}\left(c_{x}\right) .
$$

THEOREM 8.5. For any continuous $f$ that is differentiable almost everywhere, the estimator that uses

$$
\beta^{(f, \tau, \ell)}(c):=\frac{f(c)}{\min \{1, \ell \tau\}}+\frac{f^{\prime}(c)}{\tau}
$$

is unbiased.

Proof. We separately treat the cases where $\tau \ell<1$ and $\tau \ell>1$. We first show that when $\tau \ell>1$, $\beta(c)=f(c)+f^{\prime}(c) / \tau$ are unbiased estimation coefficients.

For a key of size $w$, we have density $\tau e^{-\tau x}$ to have count of $w-x \in(0, w)$ (otherwise, the key has count 0 and the estimate is 0 ). We can write

$$
\beta(y)=\frac{e^{-\tau y}}{\tau} \frac{\partial\left(f(y) e^{\tau y}\right)}{\partial y} .
$$

Consider a key of size $w$. Its expected contribution to the estimate is

$$
\begin{aligned}
& \int_{0}^{w} \tau e^{-\tau x} \beta(w-x) d x \\
& =\int_{0}^{w} \tau e^{-\tau x} \frac{1}{\tau} \frac{-\partial\left(f(w-x) e^{\tau(w-x)}\right)}{\partial x} e^{-\tau(w-x)} d x \\
& =e^{-\tau w} \int_{0}^{w} \frac{-\partial\left(f(w-x) e^{\tau(w-x)}\right)}{\partial x} d x \\
& =e^{-\tau w}\left(-\left.f(w-x) e^{\tau(w-x)}\right|_{0} ^{w}\right) \\
& =e^{-\tau w} f(w) e^{\tau w}=f(w) .
\end{aligned}
$$

We now consider the case where $\tau<1 / \ell$, showing that

$$
\beta(c)=f(c) /(\ell \tau)+f^{\prime}(c) / \tau
$$

are unbiased estimation coefficients. For a key with frequency $w$, we have density $\tau e^{-x / \ell}$ to have count of $w-x \in(0, w)$. We write

$$
\begin{gathered}
\beta(y)=\frac{e^{-y / \ell}}{\tau} \frac{\partial\left(f(y) e^{y / \ell}\right)}{\partial y} . \\
\int_{0}^{w} \tau e^{-x / \ell} \beta(w-x) d x \\
=\int_{0}^{w} \tau e^{-x / \ell} \frac{e^{-(w-x) / \ell}}{\tau} \frac{-\partial\left(f(w-x) e^{(w-x) / \ell}\right)}{\partial x} d x \\
=e^{-w / \ell} \int_{0}^{w} \frac{-\partial\left(f(w-x) e^{(w-x) / \ell}\right)}{\partial x} d x=f(w) .
\end{gathered}
$$

Note that any continuous monotone function, including the $\operatorname{cap}_{T}$ functions, is differentiable almost everywhere and hence satisfies the requirements of the theorem. 
We upper bound the $\mathrm{CV}$ of the streaming fixed- $k \mathrm{SH}_{\ell}$ estimator (Proof is in Appendix E.2):

THEOREM 8.6. The $C V$ of the estimator $Q \overline{\left(c a p_{T}, H\right)}$ as specified in Equation (15) with coefficients (16) (from an $\mathrm{SH}_{\ell}$ sample) is upper bounded by

$$
\begin{aligned}
\left.C V\left[Q \overline{\left(\operatorname{cap}_{T}, H\right.}\right)\right] & \leq \frac{e}{e-1} \max \left\{1, \frac{\ell}{T}\right\}\left(\frac{\ell}{T}\left(1-e^{-T / \ell}\right)+\frac{T}{\ell}\right) \\
& \leq\left(\frac{\frac{e}{e-1}(1+\max \{\ell / T, T / \ell\})}{q(k-1)}\right)^{0.5} .
\end{aligned}
$$

In particular, when $\ell=\Theta(T)$, the $\mathrm{CV}$ is $O\left(q(k-1)^{-0.5}\right)$, and when $\ell=T$, the $\mathrm{CV}$ is at most

$$
\left(\frac{2 e-1}{e-1} \frac{1}{q(k-1)}\right)^{0.5} \approx 1.6\left(q(k-1)^{-0.5} .\right.
$$

For the special case of sum estimation $f(w)=w$ and when $\ell \rightarrow \infty$, the sampling scheme is the same as weighted sample-and-hold [10] and the estimation coefficients are $\beta(c)=c+\tau^{-1}$. We establish in Appendix D (Theorem D.1) that the CV is upper bounded by $(q(k-1))^{-0.5}$ (the same as the bound we get when we know the frequencies of sampled keys).

\section{MULTI-OBJECTIVE SAMPLES}

We established that from a fixed- $k \mathrm{SH}_{\ell}$ sample we can estimate well cap $\cos _{T}$ statistics when $T=\Theta(\ell)$. This means that if we are interested in estimates with statistical guarantees for cap values $T=$ $[a, b]$, it suffices to use $\mathrm{SH}_{\ell}$ samples with parameters $\ell_{i}=2^{i} a$ for $i \leq\lceil\log (b / a)\rceil$. To process a query for a $\operatorname{cap}_{T}$ statistics, we can use the $\mathrm{SH}_{\ell}$ sample with $\ell$ that is closest (within a factor of $\sqrt{2}$ ) from $T$. In particular, to estimate all cap statistics, it suffices to use samples for $\left.\left\lceil\log \left(\max _{x} w_{x} / \min _{x} w_{x}\right)\right)\right\rceil \ell$ values. Moreover, we can estimate statistics for any $f$ that is a (continuous, discrete, or combined) nonnegative linear combination of $\operatorname{cap}_{T}$ functions, using a respective sum of the estimates. For example, for $f(w):=\int_{0}^{\infty} a(T) \operatorname{cap}_{T}(w) d T$ where $a(w) \geq 0$ we have

$$
Q(f, H)=\int_{0}^{\infty} a(T) Q\left(\operatorname{cap}_{T}, H\right) d T
$$

and use

$$
\widehat{Q(f, H)} \leftarrow \int_{0}^{\infty} a(T) Q\left(\overline{\operatorname{cap}_{T}, H}\right) d T .
$$

Note that since $Q \overline{\left(\operatorname{cap}_{T}, H\right)}$ are unbiased, the estimator $\widehat{Q(f, H)}$ is also unbiased (expectation of sum is sum of expectations). Moreover, the $\mathrm{CV}$ bounds for the estimates $Q \overline{\left(\operatorname{cap}_{T}, H\right)}$ also carry over to $\overline{Q(f, H)}$.

LEMMA 9.1.

$$
\left.C V[\widehat{Q(f, H)}] \leq \max _{T \mid a(T)>0} C V\left[Q \overline{\left(\operatorname{cap}_{T}, H\right.}\right)\right]
$$

Proof. We first bound the variance (using properties of the variance of a sum of random variables):

$$
\begin{aligned}
& \operatorname{var}[\overline{Q(f, H)}]=\int_{0}^{\infty} \int_{0}^{\infty} a\left(T_{1}\right) a\left(T_{2}\right) \operatorname{cov}\left[Q \overline{\left(\operatorname{cap}_{T_{1}}, H\right)}, Q \overline{\left(\operatorname{cap}_{T_{2}}, H\right)}\right] d T_{1} d T_{2} \\
& \leq \int_{0}^{\infty} \int_{0}^{\infty} a\left(T_{1}\right) a\left(T_{2}\right) \sqrt{\operatorname{var}\left[Q\left(\overline{\operatorname{cap}_{T_{1}}, H}\right)\right] \operatorname{var}\left[Q\left(\overline{\left.\operatorname{cap}_{T_{2}}, H\right)}\right]\right.} d T_{1} d T_{2} \\
& =\left(\int_{0}^{\infty} a(T) \sqrt{Q\left(\overline{\operatorname{cap}_{T}, H}\right)} d T\right)^{2} \text {. }
\end{aligned}
$$


We can now bound the $\mathrm{CV}$ :

$$
\begin{aligned}
\mathrm{CV}[\widehat{Q(f, H)}] & =\frac{\sqrt{\operatorname{var}[\widehat{Q(f, H)}]}}{Q(f, H)}=\frac{\int_{0}^{\infty} a(T) \sqrt{\left.Q \overline{\left(\operatorname{cap}_{T}, H\right.}\right)} d T}{\int_{0}^{\infty} a(T) Q\left(\operatorname{cap}_{T}, H\right) d T} \\
& \leq \max _{T \mid a(T)>0} \frac{\sqrt{Q\left(\overline{\operatorname{cap}_{T}, H}\right)}}{Q\left(\operatorname{cap}_{T}, H\right)}=\max _{T \mid a(T)>0} \mathrm{CV}\left[Q\left(\overline{\operatorname{cap}_{T}, H}\right)\right] .
\end{aligned}
$$

We now improve over this basic approach by instead of working with a set $\left\{S_{\ell}\right\}$ of samples with respective caps $\ell \in L$, we work with a single sample $S_{L}=\bigcup_{\ell \in L} S_{\ell}$. The improvement has several components: Sample coordination, which ensures that samples with closer $\ell$ are more similar so that $\left|S_{L}\right| \ll k|L|$, using estimators that benefit from the combined sample, and sampling algorithms that use structure size that is proportional to $\left|S_{L}\right|$.

\subsection{Sample Coordination}

We coordinate the samples for different $\ell[3,15]$ by using the same "randomization." In our context, the randomization of each key constitutes of two independent random variables $\operatorname{Hash}(x) \sim U[0,1]$ and $y_{x} \sim \operatorname{Exp}\left[w_{x}\right]$, which is the minimum over elements with key $x$ of the $\operatorname{Exp}[w]$ component used for scoring elements $(x, w)$. With coordination, we can express the seed of $x$ as a function of $\ell$ as

$$
\operatorname{seed}_{\ell}(x)=y_{x} \leq 1 / \ell ? \operatorname{Hash}(x) / \ell: y_{x} .
$$

The sample $S_{\ell}$ includes the $k$ keys with smallest seed $\ell$ values and its threshold $\tau_{\ell}$ is the $(k+1)$ st smallest $\operatorname{seed}_{\ell}$ value (or $+\infty$ if there are fewer than $k+1$ active keys).

Surprisingly perhaps, we show that the expected number of distinct keys in $S_{L}$ for $L=(0, \infty)$ when the samples $S_{\ell}$ are coordinated is at most $k \ln n$, where $n$ is the number of active keys in the dataset.

Lemma 9.2. Let $\left\{S_{\ell}\right\}$ for $\ell \in L=(0, \infty)$ be coordinated fixed-k $S H_{\ell}$ samples. Then $E\left[\left|S_{L}\right|\right]=$ $E\left[\left|\cup_{\ell>0} S_{\ell}\right|\right]<k \ln n$. Moreover, for $a>1$, the probability of $\left|S_{L}\right|>a k \ln n$ decreases exponentially with $a$.

Proof. Consider the set of keys ordered by increasing $y_{x}$. The sample $S_{\ell}$ is as follows. If the number of keys with $y_{x} \leq 1 / \ell$ is at most $k$, then the sample contains the first $k$ keys in the order. If there are more than $k$ keys with $y_{x} \leq 1 / \ell$, then the sample includes the $k$ among them with $\operatorname{smallest} \operatorname{Hash}(x)$ values.

For each key in the sorted order we compute the probability that it participates in $S_{\ell}$ for some $\ell$. The first $k$ keys participate in samples with large $\ell$ and hence they participate with probability 1 .

The $i$ th key $(i>k)$ can participate in a sample only if it has one of the $k$ smallest Hash $(x)$ values in any prefix of this order of length at least $i$. It therefore suffices to look at the smallest prefix that includes our key, which is of length exactly $i$. Recall that the Hash $(x)$ values are independent of $y_{x}$; hence, the probability of that event is $k / i$. Moreover, the probabilities are independent for different positions in the sequence.

The expected size $\left|S_{L}\right|$ is thus the expected number of keys that participate in at least one sample. This is equal to the sum over positions $i=1, \ldots, n$ of the probabilities that is 1 for $i \leq k$ and $k / i$ for $i \geq k$.

$$
\mathrm{E}\left[\left|S_{L}\right|\right]=\sum_{i=1}^{n} \min \{1, k / i\} \leq k \ln n .
$$

Concentration follows by noting independence of inclusions and applying Chernoff bounds. 
A corollary of the proof is that after fixing the randomization, then for each key $x$, the membership $x \in S_{\ell}$ holds for a contiguous interval of $\ell$ values $\left(a_{x}, b_{x}\right]$, where the endpoints have the general form $1 / y_{z}$ for some key $z$. If $x$ is such that $y_{x}$ is among the $k$ smallest among $\left\{y_{z} \mid z \in \mathcal{X}\right.$, then $b_{x}=+\infty$ and it $\operatorname{Hash}(x)$ is among the $k$ smallest in $\{\operatorname{Hash}(z) \mid z \in \mathcal{X}\}$ then $a_{x}=0$.

\subsection{Estimation}

We now consider estimators that leverage all the sampled keys $x \in S_{L}$ [15]. This allows us to obtain tighter estimates than when using any one sample $S_{\ell}$. We consider two-pass sampling, so that $w_{x}$ is available for sampled keys, and compute for each key $x$ a probability $\Phi\left(w_{x}\right)$ that it is included in at least one of $S_{\ell}$ for $\ell \in L$, when fixing the randomization on $X \backslash\{x\}$.

Once we have the probabilities $\Phi\left(w_{x}\right)$ we can apply an inverse probability estimate $\overline{Q(f, H)}=$ $\sum_{x \in H} f\left(w_{x}\right) / \Phi\left(w_{x}\right)$. Since $\Phi\left(w_{x}\right)$ is at least as large as the inclusion probability of $x$ in any individual $S_{\ell}$, the variance of the estimate for any query is at most that obtained by using any single sample. Also note that the estimate can still be decomposed to have the form of a sum of of $Q \overline{\left(\operatorname{cap}_{T}, H\right)}$ estimates. The variance and hence the $\mathrm{CV}$ of these estimates is at most that we would obtain with a dedicated sample. Hence, using Lemma 9.2, the CV of our estimate for $f$ is at most that of our dedicated estimators for $Q\left(\operatorname{cap}_{T}, H\right)$.

We now provide details on how we compute the probabilities $\Phi$. This probability can be computed from $w_{x}$ and the set of pairs $\left\{\ell, \tau_{\ell}^{-x}\right\}$ for $\ell \in L$. Here, we define $\tau_{\ell}^{-x}$ to be the $k$ th smallest $\operatorname{seed}_{\ell}(z)$ for $z \in X \backslash\{x\}$. When $x \in S_{\ell}$, this is the threshold $\tau_{\ell}$ and otherwise, it is $\max _{z \in S_{\ell}} \operatorname{seed}_{\ell}(z)$.

LEMMA 9.3.

$$
\Phi(x)=\operatorname{Pr}_{y \sim \operatorname{Exp}\left[w_{x}\right], h \sim U[0,1]}[\exists \ell \in L, C(\ell, x)],
$$

where $C(\ell, x)$ is the condition $y<\max \left\{\tau_{\ell}^{-x}, 1 / \ell\right\}$ and $h<\ell \tau_{\ell}^{-x}$.

Proof. Using the independent random variables $y_{x} \sim \operatorname{Exp}\left[w_{x}\right]$ and $\operatorname{Hash}(x)$, the condition for inclusion of $x$ in $S_{\ell}$ is that

$$
y_{x}<\max \left\{\tau_{\ell}^{-x}, 1 / \ell\right\} \text { and } \operatorname{Hash}(x)<\ell \tau_{\ell}^{-x} .
$$

The condition for inclusion in $S_{L}$ is that $\left(y_{x}\right.$, Hash $\left.(x)\right)$ satisfy the condition for at least one $\ell \in$ L.

When working with $L$ that contains a contiguous interval, such as $L=(0, \infty)$, we can express the $k$ th and $(k+1)$ st smallest values in $\operatorname{seed}_{\ell}(z)$ as a function of $1 / \ell$ as a piecewise linear function with at most $k \ln n$ pieces (in expectation). This allows us to compute $\Phi\left(w_{x}\right)$ for all keys in $x \in S_{L}$.

\subsection{Sampling Algorithm}

We can realize the sampling scheme with structure size that is proportional to the number of distinct keys in $\left|S_{L}\right|$. Let $\ell_{i}$ be our list of cap parameters in decreasing order. We maintain for each $i$, the $k+1$ keys with smallest Hash $(x)$ among those with $y_{x}<1 / \ell_{i}$. If for the highest $\ell$ values in $L$ there are fewer than $k+1$ keys with $y_{x}<1 / \ell$, then we include the $k+1$ keys with smallest $y_{x}$.

When $L=(0,+\infty)$ then we keep all keys with $\operatorname{Hash}(x)$ that is one of the $(k+1)$ smallest among all keys with at most the $y$ value. Note that we can do this with a composable sketch over the unagregated data. The " $y$ " value of each key is the smallest over processed elements. In expectation we keep at most $k \ln n$ keys at any point.

\section{SIMULATIONS}

Our experimental evaluation is aimed to understand the error distribution of our estimators. Our analysis provided statistical guarantees on the errors that are close to the "gold standard" 
attainable on aggregated data. The analysis, however, is worst case in terms of the dependence on the disparity $\max \{\ell / T, T / \ell\}$ and the factors of $(e /(e-1))^{0.5} \approx 1.26$ (two-pass) and $(2 e /(e-1))^{0.5} \approx 1.8$ (one-pass), which assume a worst-case frequency distribution (error is larger when $w_{x} \approx \ell$ ) and not reflecting the advantage of with-replacement sampling that is significant when there is skew. We therefore expect actual errors to be much lower than our upper bounds.

Our sampling algorithms and estimators were implemented in Python using numpy.random and hashlib libraries. Simulations were performed on MacBook Air and Mac mini computers. We did not attempt to benchmark performance in terms of running time, since computationally, our algorithms are similar to the widely applied distinct sampling or counting algorithms and can easily be tuned and scaled to very large datasets and common platforms.

We generated streams of $10^{5}$ elements with uniform values. The keys were drawn from a Zipf distribution with parameter $\alpha=1,1.1 .2,1.5,1.8,2$. This range of Zipf parameters is typical to large datasets and working with them allowed us to finely understand the error dependence on the skew (Zipf with larger $\alpha$ is more skewed and has fewer distinct keys per number of elements). The average number of distinct keys in our simulations, and respective sample sizes we used, was $4.3 \times 10^{4}$ for $\alpha=1.1$ (used $k=100$ ); $1.84 \times 10^{4}$ for $\alpha=1.2$ (used $k=100$ ); $3.04 \times 10^{3}$ for $\alpha=1.5$ (used $k=100$ ); 841 for $\alpha=1.8$ (used $k=50$ ); and 437 for $\alpha=2$ (used $k=50$ ).

For each stream, we computed the exact frequencies of each key for reference in the error computation of the estimates. For a set of sample cap parameters $\ell=1,5,20,50,100,1,000,10,000$ (and also $\ell=0.1$ with continuous samples), we computed discrete and continuous fixed- $k \mathrm{SH}_{\ell}$ samples. Discrete $\mathrm{SH}_{\ell}$ sampling used Algorithm 4 with scoring function (7) and continuous $\mathrm{SH}_{\ell}$ sampling used Algorithm 6.

From each sample, we computed an estimate of the frequency cap statistic $Q\left(\operatorname{cap}_{T}, \mathcal{X}\right)$ over all keys for parameters $T=1,5,20,50,100,1,000,10,000$. With discrete $\mathrm{SH}_{\ell}$, we used the estimator of the form (8) and computed estimation coefficients as in Theorem 7.1. With continuous $\mathrm{SH}_{\ell}$, we used the estimator (15) with coefficient function (16), which for $\operatorname{cap}_{T}$ statistics is

$$
\beta(c)=\frac{\min \{T, c\}}{\min \{1, \ell \tau\}}+\tau^{-1} I_{c<T}
$$

For each $\ell, T$ combination, we also computed the estimate that is obtained from two-pass algorithms (Section 4), applied with element scoring (Equation (7)) for discrete schemes and Equation (12) for continuous schemes. We used the inverse probability estimate $\sum_{x} \min \left\{T, w_{x}\right\} / \Phi\left(w_{x}\right)$, where $\Phi\left(w_{x}\right)$ is (13) for continuous schemes and as outlined in Section 7 for discrete schemes.

For each of these estimates, we computed the relative and NRMSE errors, averaged over multiple $(r e p=200$ or $r e p=500)$ simulations (each using a fresh hash function and randomness). Selected simulation results showing the errors for $\ell, T$ combinations are provided in Figure 3 for discrete $\mathrm{SH}_{\ell}$ and in Figure 4 for continuous $\mathrm{SH}_{\ell}$. The minimum error for each statistics $T$ across samples $\ell$ is in bold.

\subsection{Discussion of Results}

When looking at the parameter $\ell$ with smallest error for each cap statistics $T$, we see the diagonal pattern expected from our analysis, where the error is minimized when $\ell \approx T$ and degrades with disparity between $T$ and $\ell$. Note that the smallest distinct sampling threshold we had was $\tau \approx 0.001$ (for $\alpha=1.1$ ), therefore, our high $\ell$ values effectively emulated uncapped $\mathrm{SH}$.

Even for these realistic distributions, we observe that a considerable performance gain by using an appropriate sample for our particular cap statistics. We can also see that the sensitivity of the error to the parameter $\ell$ increases with skew (higher Zipf parameter $\alpha$ ). In particular, the ratio of the error to the bold minimum when using a high $\ell$ sample to estimate distinct counts was 


\begin{tabular}{|c|c|c|c|c|c|c|c|c|c|c|c|c|c|c|c|c|c|}
\hline \multicolumn{9}{|c|}{ discrete $k=100, \alpha=1.2, m=10^{5}, r e p=200$, NRMSE 1-pass } & \multicolumn{9}{|c|}{ discrete $k=100, \alpha=1.2, m=10^{5}, r e p=200$, NRMSE 2-pass } \\
\hline 5 & 0.094 & 0.093 & 0.112 & 0.144 & 0.184 & 0.332 & 0.449 & 1.316 & 5 & 0.094 & 0.093 & 0.110 & 0.143 & 0.183 & 0.333 & 0.449 & 1.316 \\
\hline 20 & 0.133 & 0.111 & 0.102 & 0.109 & 0.122 & 0.199 & 0.254 & 0.615 & 20 & 0.123 & 0.109 & 0.101 & 0.108 & 0.120 & 0.199 & 0.254 & 0.614 \\
\hline 100 & 0.146 & 0.125 & 0.104 & 0.099 & 0.099 & 0.111 & 0.133 & 0.311 & 100 & 0.144 & 0.122 & 0.105 & 0.099 & 0.097 & 0.109 & 0.131 & 0.310 \\
\hline 500 & 0.171 & 0.135 & 0.123 & 0.112 & 0.110 & 0.102 & 0.101 & 0.149 & 500 & 0.156 & 0.130 & 0.120 & 0.114 & 0.110 & 0.101 & 0.099 & 0.147 \\
\hline 1000 & 0.174 & 0.156 & 0.141 & 0.125 & 0.118 & 0.100 & 0.094 & 0.090 & 1000 & 0.161 & 0.148 & 0.137 & 0.126 & 0.118 & 0.099 & 0.092 & 0.088 \\
\hline 10000 & 0.178 & 0.148 & 0.128 & 0.110 & 0.102 & 0.083 & 0.076 & 0.056 & 10000 & 0.165 & 0.140 & 0.120 & 0.107 & 0.099 & 0.082 & 0.075 & 0.054 \\
\hline 1 & 0.102 & 0.133 & 0.193 & 0.267 & 0.330 & 0.556 & 0.700 & 1.448 & 1 & 0.102 & 0.133 & 0.193 & 0.267 & 0.330 & 0.556 & 0.700 & 1.448 \\
\hline 5 & 0.114 & 0.097 & 0.118 & 0.148 & 0.181 & 0.312 & 0.396 & 0.862 & 5 & 0.110 & 0.096 & 0.116 & 0.147 & 0.180 & 0.312 & 0.397 & 0.862 \\
\hline 20 & 0.153 & 0.111 & 0.094 & 0.101 & 0.115 & 0.183 & 0.230 & 0.5 & 20 & 139 & 0.109 & 0.094 & 0.099 & 0.1 & 0.182 & 0.230 & 0.508 \\
\hline 50 & 0.184 & 0.137 & 0.112 & 0.100 & 0.099 & 0.128 & 0.156 & 0.353 & 50 & 0.163 & 0.129 & 0.108 & 0.100 & 0.099 & 0.127 & 0.156 & 0.353 \\
\hline 100 & 0.192 & 0.141 & 0.106 & 0.091 & 0.084 & 0.097 & 0.114 & 0.240 & 100 & 0.175 & 0.133 & 0.102 & 0.088 & 0.083 & 0.097 & 0.115 & 0.240 \\
\hline 500 & 0.225 & 0.157 & 0.122 & 0.101 & 0.087 & 0.064 & 0.057 & 0.058 & 500 & 0.198 & 0.148 & 0.115 & 0.097 & 0.086 & 0.062 & 0.054 & 0.057 \\
\hline 1000 & 0.221 & 0.160 & 0.119 & 0.098 & 0.086 & 0.060 & 0.049 & 0.029 & 1000 & 0.191 & 0.144 & 0.115 & 0.097 & 0.084 & 0.056 & 0.045 & 0.024 \\
\hline 10000 & 0.223 & 0.171 & 0.127 & 0.103 & 0.091 & 0.061 & 0.049 & 0.025 & 10000 & 0.207 & 0.154 & 0.120 & 0.102 & 0.089 & 0.058 & 0.047 & 0.023 \\
\hline 50 & 0.302 & 0.191 & 0.143 & 0.126 & 0.126 & 0.165 & 0.198 & & 50 & 0.270 & 0.186 & 0.141 & 0.125 & 0.124 & 0.163 & 0.197 & 0.385 \\
\hline 100 & 327 & 0.206 & 0.154 & 0.126 & 0. & & & & 100 & & 0.187 & 0.143 & 0.123 & 0.1 & 0.1 & 0.140 & 0.276 \\
\hline 500 & 0.397 & 0.252 & 0.181 & 0.150 & 0.125 & 0.0 & 0.069 & & 500 & 16 & 0.225 & 0.172 & 0.144 & 0.123 & 0.076 & 0.064 & 0.064 \\
\hline 1000 & 0.404 & 0.258 & 0.168 & 0.137 & 0.116 & 0.0 & 0.0 & & 1000 & 0.347 & 0.231 & 0.165 & 0.134 & 0.1 & 0.0 & 0.050 & 0.021 \\
\hline 10000 & 0.416 & 0.272 & 0.181 & 0.145 & 0.112 & 0.064 & 0.049 & 0.020 & 10000 & 0.365 & 0.235 & 0.170 & 0.133 & 0.108 & 0.061 & 0.045 & 0.018 \\
\hline \multicolumn{18}{|c|}{ discrete $k=50, \alpha=2, m=10^{5}, r e p=500$, NRMSE 1-pass } \\
\hline$\ell, T$ & 1 & 5 & 20 & 50 & 100 & 500 & 1000 & 10000 & $\ell, T$ & 1 & 5 & 20 & 50 & 100 & 500 & 1000 & 10000 \\
\hline 1 & 0.145 & 0.172 & 0.235 & 0.290 & 0.345 & 0.505 & 0.601 & 1.063 & 1 & 0.145 & 0.172 & 0.235 & 0.290 & 0.345 & 0.505 & 0.601 & 1.063 \\
\hline 5 & & 0.134 & 147 & 0.170 & & & & & 5 & 0.165 & 0.132 & 0.145 & 0.169 & 0.20 & 0.311 & 0.370 & 0.636 \\
\hline 20 & 0.243 & 0.153 & 0.123 & 0.126 & 0.138 & 0.196 & 0.232 & 0.421 & 20 & 0.205 & 0.147 & 0.122 & 0.125 & 0.137 & 0.196 & 0.232 & 0.422 \\
\hline 50 & & & 0.120 & & & & 0.160 & & 50 & & 0.165 & 0.120 & 0.104 & 0.101 & 0.132 & 0.158 & 0.282 \\
\hline 100 & 0.343 & 0.211 & 0.146 & 0.116 & 0.099 & 0.097 & 0.107 & 0.185 & 100 & 0.294 & 0.194 & 0.140 & 0.112 & 0.097 & 0.094 & 0.105 & 0.184 \\
\hline 500 & & & & & & & & & 500 & & 0.202 & 0.138 & 0.105 & 0.082 & 0.042 & 0.031 & 0.016 \\
\hline 1000 & 0.384 & 0.243 & 0.156 & 0.110 & 0.086 & 0.047 & 0.036 & 0.013 & 1000 & 0.334 & 0.216 & 0.146 & 0.109 & 0.084 & 0.041 & 0.029 & 0.010 \\
\hline
\end{tabular}

Fig. 3. Simulation results for discrete $\mathrm{SH}_{\ell}$.

up to a factor of 3, whereas the reverse could be 30 -fold or more. The increase in error for midcap statistics by using the better one of $\ell=1, \infty$ instead of the minimum was up to $40 \%$. Note, however, that even this is optimistic, as we measured error on the whole population-on segments with frequency distributions that do not match that of the population, error can be much higher. ${ }^{6}$

Comparing the error of two-pass versus streaming estimates (both are the same for distinct counts $\ell=1$ but diverge otherwise), we observe that the benefit of the second pass is limited to $10 \%$ and typically lower. This agrees with our CV upper bounds that are only slightly larger for the streaming estimates. This suggests that the choice of scheme should depend on the computational platform.

The $\ell=1, T=1$ estimates have NRMSE $\approx 1 / \sqrt{k-2}$, this is because the upper bounds for approximate distinct counting are fairly tight $[5,6]$ as there is no dependence on the frequency distribution. In our simulations, for higher cap values $T$, the minimum error (over $\ell$ ) was typically much lower than the CV upper bounds. This suggests using adaptive confidence bounds, based on sampled frequencies, rather than relying only on the CV upper bounds.

\section{RELATED WORK}

There is a large body of work on computing statistics over unaggregated data that we can not hope to cover here. The toolbox includes deterministic structures such as Misra Gries for frequent keys [32], other sampling algorithms [11], and random linear projections (linear sketches) [1, 16,

\footnotetext{
${ }^{6}$ To make this point clearer, our selected segment was the whole population, which means that for the segment, the number of samples was the same as when sampling using $\ell=T$. Estimation quality deterioration from disparity was only due to the allocation of sampling probabilities within segment. We can expect worst results (but again, theory bounds the worst case pretty tightly), when adversely selecting segments. For example, sampling a skewed distribution with very large $\ell$ and choosing segments with small $w_{x}=1$ and $T=1$. In this case, the segment can have a high fraction of distinct keys but a small fraction of total frequency and will obtain very few samples.
} 


\begin{tabular}{|c|c|c|c|c|c|c|c|c|c|c|c|c|c|c|c|c|c|}
\hline$\ell, T$ & 1 & 5 & 20 & 50 & 100 & 500 & 1000 & 10000 & $\ell, T$ & 1 & 5 & 20 & 50 & 100 & 500 & 1000 & 10000 \\
\hline 0.1 & 0.098 & 0.118 & 0.180 & 0.252 & 0.326 & 0.648 & 0.897 & 2.399 & 0.1 & 0.098 & 0.118 & 0.180 & 0.252 & 0.326 & 0.649 & 0.897 & 2.399 \\
\hline 1 & 0.098 & 0.108 & 0.150 & 0.207 & 0.267 & 0.541 & 0.781 & 2.006 & 1 & 0.097 & 0.107 & 0.149 & 0.206 & 0.266 & 0.540 & 0.780 & 2.005 \\
\hline 5 & 0.109 & 0.100 & 0.110 & 0.135 & 0.170 & 0.316 & 0.432 & 1.135 & 5 & 0.106 & 0.100 & 0.111 & 0.135 & 0.171 & 0.316 & 0.433 & 1.135 \\
\hline 20 & 0.114 & 0.106 & 0.105 & 0.112 & 0.126 & 0.198 & 0.252 & 0.672 & 20 & 0.112 & 0.106 & 0.104 & 0.111 & 0.125 & 0.197 & 0.251 & 0.671 \\
\hline 50 & 0.117 & 0.106 & 0.103 & 0.105 & 0.108 & 0.145 & 0.179 & 0.418 & 50 & 0.112 & 0.106 & 0.103 & 0.103 & 0.108 & 0.144 & 0.178 & 0.418 \\
\hline 100 & 0.125 & 0.112 & 0.103 & 0.101 & 0.101 & 0.114 & 0.133 & 0.285 & 100 & 0.120 & 0.111 & 0.103 & 0.101 & 0.100 & 0.113 & 0.131 & 0.285 \\
\hline 500 & 0.141 & 0.130 & 0.122 & 0.119 & 0.115 & 0.106 & 0.103 & 0.120 & 500 & 0.137 & 0.128 & 121 & 0.117 & 0.114 & 0.105 & 0.103 & 0.120 \\
\hline 1000 & 0.144 & 0.133 & 0.123 & 0.118 & 0.112 & 0.102 & 0.097 & 0.083 & 1000 & 0.138 & 0.130 & 0.121 & 0.115 & 0.111 & 0.101 & 0.097 & 0.082 \\
\hline 10000 & 0.133 & 0.121 & 0.115 & 0.110 & 0.108 & 0.098 & 0.094 & 0.080 & 10000 & 0.130 & 0.119 & 0.112 & 0.109 & 0.106 & 0.097 & 0.093 & 0.079 \\
\hline \multicolumn{9}{|c|}{ continuous $k=100, \alpha=1.2, m=100000, r e p=500$, NRMSE 1-pass } & \multicolumn{9}{|c|}{ continuous $k=100, \alpha=1.2, m=100000, r e p=500$, NRMSE 2-pass } \\
\hline$\ell, T$ & 1 & 5 & 20 & 50 & 100 & 500 & 1000 & 10000 & $\ell, T$ & 1 & 5 & 20 & 50 & 100 & 500 & 1000 & 10000 \\
\hline 0.1 & 0.099 & 0.126 & 0.189 & 0.267 & 0.348 & 0.676 & 0.942 & 3.061 & 0.1 & 0.097 & 0.125 & 0.189 & 0.267 & 0.348 & 0.677 & 0.942 & 3.061 \\
\hline 1 & 0.099 & 0.111 & 0.161 & 0.225 & 0.291 & 0.565 & 0.788 & 2.190 & 1 & 0.097 & 0.111 & 0.162 & 0.225 & 0.291 & 0.565 & 0.787 & 2.190 \\
\hline 5 & 0.109 & 0.100 & 0.111 & 0.142 & 0.182 & 0.336 & 0.444 & 1.070 & 5 & 0.106 & 0.099 & 0.110 & 0.141 & 0.181 & 0.336 & 0.443 & 1.070 \\
\hline 20 & 0.122 & 0.105 & 0.094 & 0.102 & 0.118 & 0.202 & 0.261 & 0.649 & 20 & 0.119 & 0.102 & 0.093 & 0.101 & 0.119 & 0.203 & 0.262 & 0.650 \\
\hline 50 & 0.150 & 0.127 & 0.115 & 0.111 & 0.111 & 0.148 & 0.1 & 0.416 & 50 & 0.145 & 0.125 & 0.114 & 0.110 & 0.111 & 0.146 & 0.185 & 0.415 \\
\hline 100 & 0.153 & 0.128 & 0.116 & 0.106 & 0.102 & 0.117 & 0.137 & 0.293 & 100 & 0.147 & 0.127 & 0.113 & 0.105 & 0.102 & 0.114 & 0.135 & 0.293 \\
\hline 500 & 0.161 & 0.139 & & & & & & & 500 & 0.152 & 0.134 & & 0.115 & & 0.092 & 0.085 & 0.080 \\
\hline 1000 & 0.173 & 0.145 & 0.125 & 0.117 & 0.111 & 0.093 & 0.085 & & 1000 & 0.161 & 0.138 & 0.123 & 0.114 & & 0.092 & 0.084 & 0.060 \\
\hline 10000 & 0.169 & 0.142 & 0.125 & 0.113 & 0.106 & 0.087 & 0.079 & 0.059 & 10000 & 0.159 & 0.138 & 0.120 & 0.110 & 0.103 & 0.087 & 0.079 & 0.057 \\
\hline \multicolumn{9}{|c|}{ continuous $k=100, \alpha=1.5, m=100000, r e p=500$, NRMSE 1-pass } & \multicolumn{9}{|c|}{ continuous $k=100, \alpha=1.5, m=100000, r e p=500$, NRMSE 2-pass } \\
\hline$\ell, T$ & 1 & 5 & 20 & 50 & 100 & 500 & 1000 & 10000 & $\ell, T$ & 1 & 5 & 20 & 50 & 100 & 500 & 1000 & 10000 \\
\hline 0.1 & 0.106 & 0.134 & 0.198 & 0.266 & 0.341 & 0.558 & 0.688 & & 0.1 & 0.106 & 0.134 & 0.198 & 0.266 & 0.341 & 0.558 & 0.6 & 1.508 \\
\hline 1 & 0.103 & 0.120 & 0.168 & 0.228 & 0.29 & & & & 1 & 0.101 & 0.118 & 167 & 0.228 & & 0.478 & & 1.320 \\
\hline 5 & 0.119 & 0.096 & 0.113 & 0.142 & 0.174 & 0.301 & 0.3 & 0.76 & 5 & 0.113 & 0.096 & 111 & 0.140 & 0.1 & 0.300 & 0.3 & 0.766 \\
\hline 20 & 0.152 & & 096 & 0.100 & & & & & 20 & 0.142 & 0.110 & 095 & 0.098 & 0.110 & 0.175 & 0.2 & 0.454 \\
\hline 50 & 0.190 & & & 0.092 & 0.092 & & 0.1 & & 50 & 0.168 & 126 & 0 & 0.091 & & 0.120 & 0.1 & 0.294 \\
\hline 100 & 0.214 & & & 0.092 & 0.082 & & & & 100 & 0.191 & 0.139 & 109 & 0.091 & 082 & 0.076 & 0.0 & 0.167 \\
\hline 500 & 0.2 & & & & & & & & 500 & 0.203 & & & & & & & 0.022 \\
\hline 1000 & 0.224 & 0.163 & 0.122 & 102 & & & & 0.02 & 1000 & 0.198 & 146 & & 099 & 86 & 0.056 & 0.045 & 0.022 \\
\hline 10000 & 0.230 & 0.162 & 0.130 & 0.108 & 0.091 & 0.059 & 0.049 & 0.025 & 10000 & 0.205 & 0.152 & 0.122 & 0.104 & 0.089 & 0.057 & 0.046 & 0.023 \\
\hline \multicolumn{9}{|c|}{ continuous $k=50, \alpha=1.8, m=100000, r e p=500$, NRMSE 1-pass } & \multicolumn{9}{|c|}{ continuous $k=50, \alpha=1.8, m=100000, r e p=500$, NRMSE 2-pass } \\
\hline$\ell, T$ & 1 & 5 & 20 & 50 & 100 & 500 & 1000 & 10000 & $\ell, T$ & 1 & 5 & 20 & 50 & 100 & 500 & 1000 & 10000 \\
\hline 0.1 & 0.135 & 179 & 0.254 & 0.328 & 0.393 & & & & 0.1 & $\mathbf{0 . 1 3 4}$ & 0.179 & 0.254 & 0.328 & & & & 1.403 \\
\hline 1 & 0.142 & 0.161 & 0.220 & 0.284 & & & & & 1 & 0.142 & 0.160 & & 0.284 & & 0.5 & 0.6 & 1.166 \\
\hline 5 & & & & & & & & & 5 & & & & & & & & 0.782 \\
\hline 20 & 0.256 & & & & & & & & 20 & 0.219 & 156 & & 134 & & 0.2 & 0.2 & 0.498 \\
\hline 50 & & & & & & & & & 50 & & & & & & & & 0.325 \\
\hline 100 & 0.368 & & & & & & & & 100 & 0.317 & 214 & & 129 & & 0.097 & 0.1 & 0.191 \\
\hline 500 & & & & & & & & & & & & & & & & & 0.019 \\
\hline 1000 & 0.418 & & & 0.147 & & & & & 1000 & 0.353 & 0.242 & & 144 & & 0.0 & & 0.019 \\
\hline 10000 & 0.423 & 0.259 & 0.184 & 0.149 & 0.118 & 0.066 & 0.052 & 0.021 & 10000 & 0.358 & 0.239 & 0.173 & 0.139 & 0.115 & 0.064 & 0.047 & 0.018 \\
\hline \multicolumn{9}{|c|}{ continuous $k=50, \alpha=2, m=100000, r e p=500$, NRMSE 1-pass } & \multicolumn{9}{|c|}{ continuous $k=50, \alpha=2, m=100000, r e p=500$, NRMSE 2-pass } \\
\hline$\ell, T$ & 1 & 5 & 20 & 50 & 100 & 500 & 1000 & 1000 & $\ell, T$ & 1 & 5 & 20 & 50 & 100 & 500 & 1000 & 10000 \\
\hline 0.1 & 0.126 & 0.159 & 0.216 & 0.274 & 0.326 & 0.502 & 0.597 & & 0.1 & 0.125 & 0.159 & 0.216 & 0.274 & 0.326 & 0.502 & 0.597 & 1.061 \\
\hline 1 & & & & & & & & & 1 & & & & & & & & 0.908 \\
\hline 5 & 0.193 & & & 0.173 & 0.20 & & & & 5 & & 137 & & 172 & & 0.300 & 0.3 & 0.626 \\
\hline 20 & & & & & & & & & 20 & & & & & & 0.183 & 0.2 & 0.378 \\
\hline 50 & 0.339 & 0.206 & 0.14 & 0.108 & 0.094 & 0.0 & & & 50 & 0.282 & 0.184 & 3 & 106 & 0.093 & 0.094 & 0.106 & 0.181 \\
\hline 100 & & & & & & & & & 100 & & & & & & 0.041 & 0.030 & 0.020 \\
\hline 500 & 0.397 & 0.250 & 0.16 & 0.114 & 0.092 & 0.047 & 0.0 & 0.012 & 500 & 0.321 & 0.218 & 152 & 0.114 & 0.089 & 0.042 & 0.030 & 0.010 \\
\hline 1000 & & & & & & & & & 1000 & & 0.208 & & & 0.080 & 0.039 & 0.028 & 0.009 \\
\hline 10000 & 0.404 & 0.244 & 0.155 & 0.114 & 0.085 & 0.043 & 0.032 & 0.012 & 10000 & 0.326 & 0.213 & 0.147 & 0.109 & 0.084 & 0.040 & 0.028 & 0.010 \\
\hline
\end{tabular}

Fig. 4. Simulation results for continuous $\mathrm{SH}_{\ell}$.

28, 29]. Linear sketches project the key-frequency vectors to a lower dimensional vector. Linearity implies efficient updates of the sketch when processing streamed or distributed elements. Most related to frequency cap statistics are sketches based on $p$-stable distributions that are designed to estimate frequency moments for $p \in[0,1]$ [28] and $L_{p}$ sampling [30,33]. These techniques do not apply for cap statistics, as there are no appropriate stable distributions for cap functions. They are also specific to the choice of $p$ and there is no support for segment queries. $L_{p}$ samples, which sample keys roughly proportionally to $w_{x}^{p}$, are with-replacement, so less effective for skewed data, and have polylogarithmic encoding overhead. Of relevance to us is also a characterization of all monotone frequency statistics that can be estimated in polylogarithmic space and a single pass [2]. The construction, however, is mostly of theoretical interest. Generally, linear sketches have a significant encoding overhead and in practice, when updates are positive, are outperformed by sample-based sketches. In particular, all practical distinct counting algorithms are based on the sample-based MinHash sketches [6, 20, 21, 25]. For sum queries, weighted SH experimentally dominated linear sketches even in the presence of some negative updates [10].

\section{CONCLUSION}

We propose a simple but powerful bottom- $k$ sampling framework that is suitable for streamed or distributed unaggregated data. Our framework generalizes classic schemes for distinct and sum 
statistics. At the core we use a randomized scoring function of data elements that is tailored to the statistics we want to estimate. The sample then includes the keys with the lowest element scores. We specify scoring functions that are tailored for cap statistics and derive corresponding estimators that have quality that nearly matches that of a sample of the same size that is computed over the aggregated view.

Looking ahead, we would like to apply our framework to other statistics, extend it to support negative updates [10,22], and understand the theoretical limits of the approach.

\section{APPENDIXES}

\section{A BOTTOM- $K$ PROPERTIES}

Consider bottom- $k$ sampling of key value pairs $\left\{\left(x, w_{x}\right)\right\}$, where $w_{x}>0$. The sampling is specified by a continuous probability distribution SeedDist $[w]$ parametrized by $w$ with support on the nonnegative reals and such that

$$
\forall w>0 \forall \epsilon>0, \underset{x \sim \operatorname{SeedDist}[w]}{\operatorname{Pr}}[x \leq \epsilon]>0 .
$$

Each key is assigned an independent $\operatorname{seed}(x) \sim \operatorname{SeedDist}\left[w_{x}\right]$ and a bottom- $k$ sample $S$ includes the $k$ keys with smallest seed $(x)$ and also the $(k+1)$ st smallest seed $(x)$ value, which we denote by $\tau$.

We use this sample to estimate statistics $Q(f, H)$ where $f(w) \geq 0$ is such that $f(w)>0 \Rightarrow w>$ 0 . We compute per-key estimates $\overline{f\left(w_{x}\right)} \geq 0$ that are positive only when $x \in S$. We then estimate

$$
\widehat{Q(f, H)}:=\sum_{x \in \mathcal{X} \cap H} \overline{f\left(w_{x}\right)}=\sum_{x \in S \cap H} \overline{f\left(w_{x}\right)} .
$$

Some bottom- $k$ sampling schemes, as when sampling aggregated data or when performing a second pass provide us with the frequencies $w_{x}$ (and thus $f\left(w_{x}\right)$ ) for sampled keys $x \in S$, and we use the estimate

$$
\overline{f\left(w_{x}\right)}:=\frac{f\left(w_{x}\right)}{\operatorname{Pr}[\operatorname{seed}[x] \leq \tau]} .
$$

Assumptions on the streaming estimator: Our streaming schemes do not provide us with $f\left(w_{x}\right)$. Instead, when $x$ is sampled, we obtain a random variable $\overline{f\left(w_{x}\right)}$. We assume now that $\overline{f\left(w_{x}\right)}$ has the following properties:

(1) $\overline{f\left(w_{x}\right)} \sim A\left[w_{x}, \tau\right]$ from a distribution parameterized by $\tau$ and $w_{x}$ such that

$$
\mathrm{E}_{A \sim A\left[w_{x}, \tau\right]}[A]=\frac{f\left(w_{x}\right)}{\operatorname{Pr}[\operatorname{seed}[x] \leq \tau]} .
$$

(2) Conditioned on the keys included in $S$ and on the value $\tau$, the random variables $\overline{f\left(w_{x}\right)}$ for $x \in S$ are independent.

(3) The variance of the estimator when conditioned on $\tau$ is non-increasing: $\operatorname{var}\left[\overline{f\left(w_{x}\right)} \mid \tau\right]=$ $\operatorname{Pr}[\operatorname{seed}[x] \leq \tau] \mathrm{E}_{A \sim A\left[w_{x}, \tau\right]}\left[A^{2}\right]-w_{x}^{2}$ is non-increasing in $\tau$.

Note that these assumptions always hold for the inverse probability estimator (19). We next establish some useful properties that follow from these assumptions.

We first show that for all $x, \widehat{f\left(w_{x}\right)}$ is an unbiased estimate of $f\left(w_{x}\right)$. Therefore, from linearity of expectation, Equation (18) is an unbiased estimate of the statistics. We establish this using assumption (1)

LeMmA A.1 (UnBiAsEDness). $E\left[\overline{f\left(w_{x}\right)}\right]=f\left(w_{x}\right)$. 
Proof. Let $\tau$ be the $k$ th smallest seed[ $y]$ value for $y \neq x$. It is a random variable from some distribution $\tau \sim T_{x}$.

We first consider the expectation of $\overline{f\left(w_{x}\right)}$ conditioned on fixed seed $[y]$ for all $y \neq x$ (and thus fixed $\tau$ ). We have

$$
x \in S \Longleftrightarrow \operatorname{seed}[x]<\tau .
$$

Thus, using assumption (1)

$$
\mathrm{E}\left[\overline{f\left(w_{x}\right)} \mid \tau\right]=0(1-\operatorname{Pr}[\operatorname{seed}[x]<\tau])+\operatorname{Pr}[\operatorname{seed}[x]<\tau] \mathrm{E}_{A \sim A\left[w_{x}, \tau\right]}[A]=f\left(w_{x}\right) .
$$

Finally,

$$
\mathrm{E}\left[\overline{f\left(w_{x}\right)}\right]=\mathrm{E}_{\tau \sim T_{x}}\left[\mathrm{E}\left[\overline{f\left(w_{x}\right)} \mid \tau\right]\right]=\mathrm{E}_{\tau \sim T_{x}}\left[f\left(w_{x}\right)\right]=f\left(w_{x}\right) .
$$

We show that the estimates for different keys have nonpositive covariances. We will use assumption (2) that states that the estimates are independent when conditioned on $\tau$ and on inclusion of two keys.

Lemma A.2 (Nonpositive covariances). For any two keys $x, y$

$$
E\left[\overline{f\left(w_{x}\right)} \widehat{f\left(w_{y}\right)}\right] \leq E\left[\overline{f\left(w_{x}\right)}\right] E\left[\overline{f\left(w_{y}\right)}\right]=f\left(w_{x}\right) f\left(w_{y}\right) .
$$

Proof. When $k=2$, at most one of $x$ or $y$ can be sampled (there is one key in the sample). So one of the estimates must be 0 and thus $\overline{f\left(w_{x}\right)} \overline{f\left(w_{y}\right)}=0$, and we have strict inequality (negative covariance).

When $k>2$, we show that the covariance is zero. That is, $\mathrm{E}\left[\overline{f\left(w_{x}\right)} \overline{f\left(w_{y}\right)}\right]=f\left(w_{x}\right) f\left(w_{y}\right)$.

The seed values seed $[z]$ of all keys other than $x, y$ determines the value $\tau \sim T_{x y}$ of the $k-2$ smallest seed among these keys.

Consider now the distribution of $\operatorname{seed}[x]$ and seed $[y]$ (these are independent distributions) consitioned on $\tau$. The only case where $\overline{f\left(w_{x}\right)} \hat{f\left(w_{y}\right)}>0$ is when $\operatorname{seed}[x] \operatorname{seed}[y]<\tau$. The seed random variables are independent and thus the probability of this event is $\operatorname{Pr}[\operatorname{seed}[x]<$ $\tau] \operatorname{Pr}[\operatorname{seed}[y]<\tau]$.

When this event happens, the sample has threshold value $\tau$ (the $k+1$ st smallest seed amongs all keys). Conditioned on this, using assumption (2), we have that $\overline{f\left(w_{x}\right)}$ and $\overline{f\left(w_{y}\right)}$ are independent nonnegative random variables with expectation

$$
\begin{aligned}
\mathrm{E}\left[\overline{f\left(w_{x}\right)} \mid \tau, x \text { sampled }\right] & =\frac{f\left(w_{x}\right)}{\operatorname{Pr}[\operatorname{seed}[x]<\tau]} \\
\mathrm{E}\left[\overline{f\left(w_{y}\right)} \mid \tau, y \text { sampled }\right] & =\frac{f\left(w_{y}\right)}{\operatorname{Pr}[\operatorname{seed}[x]<\tau]} .
\end{aligned}
$$

Hence the expectation of the product is the product of the expectations that is

$$
\frac{f\left(w_{x}\right) f\left(w_{y}\right)}{\operatorname{Pr}[\operatorname{seed}[x]<\tau] \operatorname{Pr}[\operatorname{seed}[y]<\tau]} .
$$

In all other cases (probability $1-\operatorname{Pr}[\operatorname{seed}(x)<\tau] \operatorname{Pr}[\operatorname{seed}(y)<\tau]$ ), at most one of $x, y$ is sampled and $\overline{f\left(w_{x}\right)} \overline{f\left(w_{y}\right)}=0$.

Thus, the conditioned expectation

$$
\mathrm{E}\left[\overline{f\left(w_{x}\right)} \overline{f\left(w_{y}\right)} \mid \tau\right]=f\left(w_{x}\right) f\left(w_{y}\right) .
$$

Therefore, the unconditioned expectation is the same

$$
\mathrm{E}\left[\overline{f\left(w_{x}\right)} \widehat{f\left(w_{y}\right)}\right]=\mathrm{E}_{\tau \sim T_{x y}} \mathrm{E}\left[\widehat{f\left(w_{x}\right)} \widehat{f\left(w_{y}\right)} \mid \tau\right]=f\left(w_{x}\right) f\left(w_{y}\right) .
$$




\section{A.1 Bounding Variance}

The zero covariances property allows us to bounding the variance of our estimates of statistics by the sum of the per-key variances:

$$
\operatorname{var}[\overline{Q(f, H)}] \leq \sum_{x \in \mathcal{X} \cap H} \operatorname{var}\left[\overline{f\left(w_{x}\right)}\right]
$$

We first consider the variance of $\widehat{f\left(w_{x}\right)}$ conditioned on $\tau$, which is

$$
\begin{aligned}
\operatorname{var}\left[\overline{f\left(w_{x}\right)} \mid \tau\right] & =0^{2}(1-\operatorname{Pr}[\operatorname{seed}[x]<\tau])+\operatorname{Pr}[\operatorname{seed}[x]<\tau] \mathrm{E}_{A \sim A\left[w_{x}, \tau\right]}\left[A^{2}\right]-f\left(w_{x}\right)^{2} \\
& =\operatorname{Pr}[\operatorname{seed}[x]<\tau] \mathrm{E}_{A \sim A\left[w_{x}, \tau\right]}\left[A^{2}\right]-f\left(w_{x}\right)^{2} .
\end{aligned}
$$

The unconditioned variance depends on the distribution of $\tau$, which might be slightly different for different keys

$$
\operatorname{var}\left[\overline{f\left(w_{x}\right)}\right]=\mathrm{E}_{\tau \sim T_{x}} \operatorname{var}\left[\overline{f\left(w_{x}\right)} \mid \tau\right] .
$$

We relate the variance (20) to the conditioned per-key variance (which we can bound). We use assumption (3) that $\operatorname{var}\left[\overline{f\left(w_{x}\right)} \mid \tau\right]$ is non-increasing with $\tau$. We will also use the following notion of domination of a distributions by another distribution (or function): A distribution on $y \geq 0$ with density function $b$ is dominated by a function $s$ if

$$
\forall z, \int_{0}^{z} b(y) d y \leq \int_{0}^{z} s(y) d y .
$$

To bound Equation (20), we will specify a closed expression for a function $T$ that dominates the distributions $T_{x}$ for all $x$. We then have the relation

LemmA A.3. If $\operatorname{var}\left[\overline{f\left(w_{x}\right)} \mid \tau\right]$ is non-increasing in $\tau$ and $T_{x}$ is dominated by $T$, then $\operatorname{var}\left[\overline{f\left(w_{x}\right)}\right]=E_{\tau \sim T_{x}}\left[\operatorname{var}\left[\overline{f\left(w_{x}\right)} \mid \tau\right]\right]=\int_{0}^{\infty} T_{x}(\tau) \operatorname{var}\left[\overline{f\left(w_{x}\right)} \mid \tau\right] d \tau \leq \int_{0}^{\infty} T(\tau) \operatorname{var}\left[\overline{f\left(w_{x}\right)} \mid \tau\right] d \tau$.

The lemma enables us to bound the variance (20) of the statistics in terms of the per-key fixedthreshold variance using:

$$
\begin{aligned}
\operatorname{var}[\widehat{Q(f, H)}] & =\sum_{x \in \mathcal{X} \cap H} \operatorname{var}\left[\overline{f\left(w_{x}\right)}\right] \\
& \leq \sum_{x \in \mathcal{X} \cap H} \int_{0}^{\infty} T(\tau) \operatorname{var}\left[\overline{f\left(w_{x}\right)} \mid \tau\right] d \tau
\end{aligned}
$$

\section{B PARTIAL FREQUENCY COUNTS DISTRIBUTION}

We provide the proof of Theorem 8.4.

Proof. We first consider fixed- $\tau \mathrm{SH}_{\ell}$. We use $\Phi(w)$ (Equation (13)), which is the probability that a key of frequency $w$ is sampled. This is the same as the probability of a key with $w_{x} \gg w$ starting to get counted after processing $y \leq w$ of its occurrence frequency. The partial derivative of $\Phi(w)$ with respect to $w$ is the density function $\phi(y)$ on the frequency $y$ at which a key starts getting counted:

$$
\begin{aligned}
\phi(w) & =\frac{\partial \Phi(w)}{\partial w} \\
& =\min \{1, \tau \ell\} \max \{1 / \ell, \tau\} \exp (-w \max \{1 / \ell, \tau\} \\
& =\tau \exp (-w \max \{1 / \ell, \tau\} .
\end{aligned}
$$


For a particular key $x$, the density function of $c_{x}$ is equal to $\phi$ when in the range $\left[0, w_{x}\right]$. Elsewhere we have that $\int_{w_{x}}^{\infty} \phi(y) d y=1-\Phi\left(w_{x}\right)$ is the probability that $x$ is not sampled.

We now establish our claim for the fixed-size sampling algorithm. We start with a precise definition of the conditioning we use. The randomization used for a key $x$ includes the random hash value used in KeyBase $(x)$, the randomization used to assign scores to all the elements of $x$, and the random $u_{x}, z_{x}$ (freshly drawn per eviction step) used to adjust the counters. Observe that given that a key $x$ is cached, the threshold value $\tau$ only depends on the randomization of the other keys but not on that of $x$. When $x$ is not cached, the threshold value $\tau$ may depend on the randomization used for $x$. But in this case, $c_{x}=0$.

We show that after each step, the distribution of the final value of $c_{x}$ has the claimed density, when conditioned on the current threshold $\tau$. Correctness when $\tau$ does not change follows from the treatment of the fixed-threshold case. It remains to consider eviction steps. Let $\tau$ be the threshold value before eviction and let $\tau^{*}$ be the new threshold value after eviction. If the new $\tau^{*}$ is determined by our key $x$, then our key $x$ is evicted. Otherwise, the new $\tau^{*}$ is determined by the $k$ th largest $z_{x}$ among all other keys. This value depends only on the randomization of other keys and not on $u_{x}, r_{x}$.

We now need to show that the particular computation we used for the count adjustment preserves the claimed form of the distribution. For that we assume that the distribution of $c_{x}$ was as claimed with respect to the initial threshold $\tau$. We express it as a function of the final threshold $\tau^{*}$.

We first consider the case $\tau \ell>1$ and $\tau^{*} \ell \geq 1$. With probability $\tau^{*} / \tau$ the density at $y$ is the same and with probability $\left(1-\tau^{*} / \tau\right)$, it is the integral over $u$ of the density with $\tau$ at $u<y$ and the density of a new deduction, which is $\operatorname{Exp}\left(\tau^{*}\right)$ at $y-u$. Now observe that the density of the deduction conditioned on $\tau$ before the adjustment was (by our assumption) $\tau e^{-y \tau}$. We obtain

$$
\begin{aligned}
& \frac{\tau^{*}}{\tau} \tau e^{-\tau y}+\left(1-\frac{\tau^{*}}{\tau}\right) \int_{0}^{y} \tau e^{-\tau u} \tau^{*} e^{-\tau^{*}(y-u)} d u \\
& =\tau^{*} e^{-\tau y}+\tau^{*}\left(\tau-\tau^{*}\right) e^{-\tau^{*} y} \int_{0}^{y} e^{-u\left(\tau-\tau^{*}\right)} d u \\
& =\tau^{*} e^{-\tau y}+\tau^{*} e^{-\tau^{*} y}\left(1-e^{-y\left(\tau-\tau^{*}\right)}\right) \\
& =\tau^{*} e^{-\tau^{*} y} .
\end{aligned}
$$

We now consider the case that $\tau \ell>1$ and $\tau^{*} \ell<1$. With probability $1-\tau^{*} \ell$, we have KeyBase $(x) \geq \tau^{*}$ and the final count is 0 . With probability $(\ell \tau)^{-1} \tau^{*} \ell=\tau^{*} / \tau$, we have $u_{x} \leq$ $1 /(\ell \tau)$ and KeyBase $(x)<\tau^{*}$ and the count remains the same. Otherwise, with probability $(1-$ $\left.(\ell \tau)^{-1}\right) \tau^{*} \ell=\tau^{*}\left(\ell-\tau^{-1}\right)$ we have $u_{x}>1 /(\ell \tau)$ and KeyBase $(x)<\tau^{*}$. In this case we consider the density $y$ of the sum of the previous $u$ and new deduction $(y-u) \sim \operatorname{Exp}(1 / \ell)$. We obtain

$$
\begin{aligned}
& \frac{\tau^{*}}{\tau} \tau e^{-\tau y}+\tau^{*}\left(\ell-\tau^{-1}\right) \int_{0}^{y} \tau e^{-\tau u}(1 / \ell) e^{-(y-u) / \ell} d u \\
& =\tau^{*} e^{-\tau y}+\tau^{*}\left(\tau-\ell^{-1}\right) e^{-y / \ell} \int_{0}^{y} e^{-\left(\tau-\ell^{-1}\right) u} d u \\
& =\tau^{*} e^{-\tau y}+\tau^{*} e^{-y / \ell}\left(1-e^{-\left(\tau-\ell^{-1}\right) y}\right) \\
& =\tau^{*} e^{-y / \ell}
\end{aligned}
$$

Last, we consider the case $\tau \ell<1$. With probability $\tau^{*} / \tau$ the key maintains the same count, since conditioned on KeyBase $(x)<\tau$, we have KeyBase $(x)<\tau^{*}$ with probability $\tau^{*} / \tau$. Otherwise, the 
count is 0 . So we obtain the density

$$
\frac{\tau^{*}}{\tau} \tau e^{-y / \ell}=\tau^{*} e^{-y / \ell}
$$

\section{PPSWOR VARIANCE ANALYSIS}

We use the bottom- $k$ framework and variance analysis methodology in Appendix A to upper bound the $\mathrm{CV}$ for ppswor. Our bounds for other sampling schemes build on the ppswor proof we present here.

We use the notation $s_{W, k}$ and $S_{W, k}$, respectively, for the density and cummulative distribution functions of the Erlang distribution $\operatorname{Erlang}(W, k)$, which is a sum of $k$ independent exponential distribution with parameter $W$.

LEMma C.1. Let B be a distribution that can be expressed as the sum of $k$ independent exponential distributions, each with parameter that is at most $W$ (the set of parameters can be a random variable and parameters may not be independent). Then $B$ is dominated by $\operatorname{Erlang}(W, k)$.

Proof. The claim follows from (i) for any $W^{\prime} \leq W$, the distribution $\operatorname{Exp}\left[W^{\prime}\right]$ is dominated by $\operatorname{Exp}[W]$ (because for all $x>0,1-\exp \left(-x W^{\prime}\right) \leq 1-\exp (-x W)$. (ii) Suppose distribution $A_{i}$ is dominated by $B_{i}$. Then domination also applies to the sums: The distribution of the sum of random variables $a_{i} \sim A_{i}$ is dominated by the distribution of the sum of $b_{i} \sim B_{i}$. (Proof by simple induction).

Consider now ppswor sampling of $\mathcal{X}$ with respect to weights $w_{x}$ and let $W=\sum_{z} w_{z}$. For a particular key $x$, let $B_{x}$ be the distribution of the $k$ th smallest seed value in $\mathcal{X} \backslash x$.

Lemma C.2. For all keys $x$, the distribution $B_{x}$ is dominated by $\operatorname{Erlang}(W, k)$.

Proof. Let $\tau^{\prime} \sim B_{x}$. By definition, $\tau^{\prime}$ is the $k$ th smallest of independent exponential random variables with parameters $w_{y}$ for $y \in X \backslash x$. From properties of the exponential distribution, the minimum seed is exponentially distributed with parameter $\sum_{y \in X} \backslash x w_{y}=W-w_{x}$. Conditioned on a particular key $z_{1}$ having the smallest seed, the difference between the minimum and second smallest is exponentially distributed with parameter $W-w_{x}-w_{1}$, where $w_{1}$ is the weight of the key $z_{1}$ with minimum seed, and so on. Therefore, the distribution on $\tau^{\prime}$ conditioned on the ordered set of smallest-seed elements is a sum of $k$ exponential random variables with parameters at most $W$. The distribution $B_{x}$ is a convex combination of such distributions: One distribution for each possible ordered subset of size $k$ of $\mathcal{X} \backslash x$, and each such choice has probability equal to the probability of the ordered subset being the first $k$ keys of ppswor sampling from $\mathcal{X} \backslash x$,

Therefore, from Lemma C.1, the distribution $B_{x}$ (for any $x$ ) is dominated by Erlang with parameters $(W, k)$.

Theorem C.3. Consider ppswor sampling with respect to weights $f\left(w_{x}\right)$. For a segment $H$ with proportion $q=Q(f, H) / Q(f, \mathcal{X})$, the $C V$ of the estimate $\overline{Q(f, H)}(2)$ is at most $(q(k-1))^{-0.5}$.

Proof. We extend the analysis in References [5, 6] (note that here we take $k$ to be the sample size without the threshold ( $k+1$ smallest seed) whereas in References $[5,6] k$ is larger by 1$)$.

WLOG, since we are considering sampling aggregated data, we assume $w_{x} \equiv f\left(w_{x}\right)$. Let $W=$ $\sum_{x \in X} w_{x}$ be the total weight of the population.

We first consider the variance of the inverse probability estimate for a key with weight $w$ with respect to a fixed-threshold $\tau$. The variance is $(1 / p-1) w^{2}$, where $p=1-e^{-w \tau}$ and is at most

$$
\operatorname{var}\left[\hat{w}_{x} \mid \tau\right]=w^{2} \frac{e^{-\tau w}}{1-e^{-\tau w}} \leq w / \tau
$$

using the relation $e^{-x} /\left(1-e^{-x}\right) \leq 1 / x$. 
We now consider the "perspective" of a key $x$ and the distribution $B_{x}$ of the $k$ th smallest seed value in $\mathcal{X} \backslash x$. From Lemma C.2, $B_{x}$ is dominated by $\operatorname{Erlang}(W, k)$.

We will bound the variance of the estimate using the relation

$$
\operatorname{var}\left[\hat{w}_{x}\right]=\mathrm{E}_{\tau^{\prime} \sim B_{x}} \operatorname{var}\left[\hat{w}_{x} \mid \tau^{\prime}\right] .
$$

Since the conditioned variance $\operatorname{var}\left[\hat{w}_{x} \mid \tau^{\prime}\right]$ is non-increasing with $\tau^{\prime}$, from Lemma A.3, domination implies that

$$
\mathrm{E}_{\tau^{\prime} \sim B_{x}} \operatorname{var}\left[\hat{w}_{x} \mid \tau^{\prime}\right] \leq \mathrm{E}_{\tau^{\prime} \sim S_{W, k}} \operatorname{var}\left[\hat{w}_{x} \mid \tau^{\prime}\right]
$$

Therefore, it suffices to upper bound the expectation for $S_{W, k}$.

We now use the Erlang density function [19]

$$
s_{W, k}(x)=\frac{W^{k} x^{k-1}}{(k-1) !} e^{-W x}
$$

and the relation $\int_{0}^{\infty} x^{a} e^{-b x} d x=a ! / b^{a+1}$ to bound the variance,

$$
\begin{aligned}
\operatorname{var}\left[\hat{w}_{x}\right] & \leq \int_{0}^{\infty} s_{W, k}(z) \operatorname{var}\left[\hat{w}_{x} \mid z\right] d z \\
& \leq \int_{0}^{\infty} \frac{W^{k} z^{k-2}}{(k-1) !} e^{-W z} \frac{w}{z} d z \\
& \leq w \frac{W^{k}}{(k-1) !} \int_{0}^{\infty} z^{k-2} e^{-W z} d z=\frac{w W}{k-1} .
\end{aligned}
$$

Since covariances between different keys are nonpositive [14] (and see Appendix A) the variance on a set $H$ with weight $w(H)$ is at most the sum of variances $\operatorname{var}[\hat{w}(H)] \leq w(H) W /(k-1)$. We divide by $w(H)^{2}$ and take the square root to obtain an upper bound on the CV.

\section{CV BOUND FOR SUM STATISTICS WITH WEIGHTED SH}

We now consider the fixed- $k$ SH estimator applied for sum statistics $f(w) \equiv w$. The estimate is $\hat{Q}(w, H)=\sum_{x \in H}\left(c_{x}+\tau^{-1}\right)[10]$. Note that this is the same estimate we obtain with our streaming continuous-design estimator with $\ell=\infty$ (Section 8): The estimation coefficients are $\beta(c)=c+$ $\tau^{-1}$. Recall that the sample distribution is the same as ppswor. The streaming estimator has at least the variance of the ppswor estimator (which uses the exact frequencies as weights) since it works with less information and the ppswor inverse probability estimator minimizes variance (over all unbiased estimators that can be expressed as a sum over sampled keys of per-key unbiased estimates). Surprisingly, we obtain the same upper bound on the CV:

Theorem D.1. The weighted SH sum estimator on a segment $H$ with proportion $q=w(H) / w(X)$ has $C V$ of at most $(q(k-1))^{-0.5}$.

Proof. We bound the variance of the estimate for key $x$ conditioned on $\tau$. With probability $e^{-\tau w}$ the key is not sampled, the estimate is 0 , and the contribution to variance is $w^{2}$. Otherwise, with density $\tau e^{-\tau y}$ the count is $c_{x}=w-y$, the estimate is $c_{x}+\tau^{-1}$, and the contribution to the variance is $\left(\tau^{-1}-y\right)^{2}$. We obtain

$$
\begin{aligned}
\operatorname{var}\left[\hat{w}_{x} \mid \tau\right] & =w^{2} e^{-w \tau}+\int_{0}^{w} \tau e^{-y \tau}\left(\tau^{-1}-y\right)^{2} d y \\
& =\tau^{-2}\left(1-e^{-\tau w}\right) \leq w / \tau
\end{aligned}
$$

The last inequality uses the relation $1-e^{-x} \leq x$. 
Note that the conditioned variance bound of $w / \tau$ is the same as we obtained in the proof of Theorem C.3. Also note that the distribution of $\tau^{\prime}$, the $k$ th smallest seed in $X \backslash x$, is the same as in ppswor and we can conclude as in the proof of Theorem C.3: We take the expectation of this variance over the distribution $S_{W, k}$ that dominates $B_{x}$ and using the nonpositive covariances, bound the variance on $w(H)$ by $\frac{W w(H)}{k-1}$.

\section{E CV BOUND FOR CAP $\mathrm{P}_{T}$ statistics with $\mathrm{SH}_{\ell}$}

In this section we provide the variance analysis for our continuous-design (Section 8). We bound the variance by relating the distribution of seed $(x)$ under fixed- $k \mathrm{SH}_{\ell}$ to the distribution with ppswor with respect to key weights $\min \left\{w_{x}, T\right\}$, that is, using seed $(x) \sim \operatorname{Exp}\left[\min \left\{w_{x}, T\right\}\right]$. For the purpose of this analysis, we work with $\mathrm{SH}_{\ell}$ seed distribution that is exponential when conditioned on $y<1 / \ell$ instead of uniform. This does not change the algorithm or estimation, since there is a monotone transformation that preserves seed order. $\mathrm{The}^{\mathrm{S}} \mathrm{H}_{\ell}$ density function of the seed of a key with frequency $w$ is

$$
b_{\ell, w}(y)=(y<1 / \ell) ? \ell e^{-\ell y} \frac{1-e^{-w / \ell}}{1-1 / e}: w e^{-w y} .
$$

We now can relate $b_{\ell, w_{x}}(y)$ to $\operatorname{Exp}\left[\min \left\{w_{x}, T\right\}\right]$ :

Lemma E.1. For any key $x$ and cap value $T$, the density function $b_{\ell, w_{x}}(y)$ of $\operatorname{seed}(x)$ under fixed- $k$ $\mathrm{SH}_{\ell}$ is dominated by

$$
\frac{e}{e-1} \max \{1, \ell / T\} \min \left\{w_{x}, T\right\} e^{-\min \left\{w_{x}, T\right\} y} .
$$

Proof. We show that for any point $z \geq 0$,

$$
\begin{aligned}
& \int_{0}^{z} b_{\ell, w}(y) d y \\
& \leq \int_{0}^{z} \frac{e}{e-1} \max \left\{1, \frac{\ell}{T}\right\} \min \{w, T\} e^{-\min \{w, T\} y} d y \\
& =\frac{e}{e-1} \max \left\{1, \frac{\ell}{T}\right\}\left(1-e^{-\min \{w, T\} z}\right) .
\end{aligned}
$$

The proof is via case analysis. We start with $z \leq 1 / \ell$. We have that

$$
\begin{aligned}
\int_{0}^{z} b_{\ell, w_{x}}(y) d y & =\int_{0}^{z} \ell e^{-\ell y} \frac{1-e^{-w / \ell}}{1-e^{-1}} \\
& =\left(1-e^{-\ell z}\right) \frac{1-e^{-w / \ell}}{1-e^{-1}} .
\end{aligned}
$$

Therefore, to establish the claim (24) we need to show that

$$
\left(1-e^{-\ell z}\right)\left(1-e^{-w / \ell}\right) \leq \max \left\{1, \frac{\ell}{T}\right\}\left(1-e^{-\min \{w, T\} z}\right) .
$$

- Case $w<T$ :

$$
\left(1-e^{-\ell z}\right)\left(1-e^{-w / \ell}\right) \leq\left(1-e^{-w z}\right)=\left(1-e^{-\min \{w, T\} z}\right)
$$

using the relation

$$
\forall a, b \geq 0,\left(1-e^{-a}\right)\left(1-e^{-b}\right) \leq 1-e^{-a b} .
$$

and thus Equation (25) holds. 
- Case $w \geq T$ and $\ell \geq T$ : We have

$$
\begin{aligned}
\left(1-e^{-\ell z}\right)\left(1-e^{-w / \ell}\right) & \leq 1-e^{-\ell z} \leq \frac{\ell}{T}\left(1-e^{-T z}\right) \\
& =\frac{\ell}{T}\left(1-e^{-\min \{w, T\} z}\right) .
\end{aligned}
$$

The last inequality follows from the function $(1-\exp (-x) / x$ being monotone decreasing: Therefore $\ell \geq T$ implies $\ell z \geq T z$ and thus $\frac{\left(1-e^{-\ell z}\right)}{\ell z} \leq \frac{\left(1-e^{-T z}\right)}{T z}$ that implies $\left.1-e^{-\ell z}\right) \leq \frac{\ell}{T}(1-$ $\left.e^{-T z}\right)$. We therefore obtain that Equation (25) holds.

- Case $\boldsymbol{w} \geq T$ and $\ell \leq T$ : We have

$$
\begin{aligned}
\left(1-e^{-\ell z}\right)\left(1-e^{-w / \ell}\right) & \leq\left(1-e^{-T z}\right) \\
& =\left(1-e^{-\min \{w, T\} z}\right)
\end{aligned}
$$

and therefore Equation (25) holds.

We now consider $z \geq 1 / \ell$. We have

$$
\begin{aligned}
\int_{0}^{1 / \ell} b_{\ell, w_{x}}(y) d y & =1-e^{-w / \ell} \\
\int_{1 / \ell}^{z} b_{\ell, w_{x}}(y) d y & =e^{-w / \ell}-e^{-w z} .
\end{aligned}
$$

Thus, $\int_{0}^{z} b_{\ell, w_{x}}(y) d y=1-e^{-w z}$. To verify Equation (24), we need to show that for all $z \geq 1 / \ell$,

$$
1-e^{-w z} \leq \frac{e}{e-1} \max \left\{1, \frac{\ell}{T}\right\}\left(1-e^{-\min \{w, T\} z}\right) .
$$

This is immediate for $w \leq T$. We now consider $w \geq T$. Since $1-e^{-w z} \leq 1$, it suffices to show that

$$
\frac{e}{e-1} \max \left\{1, \frac{\ell}{T}\right\}\left(1-e^{-T z}\right) \geq 1 \text {. }
$$

Using $z \geq 1 / \ell$, it suffices to show

$$
\frac{e}{e-1} \max \left\{1, \frac{\ell}{T}\right\}\left(1-e^{-T / \ell}\right) \geq 1
$$

If $T \geq \ell$, then we substitute $1-e^{-T / \ell} \geq 1-e^{-1}=\frac{e-1}{e}$ to show that Equation (28) holds. If $T<\ell$, then we have $T / \ell<1$ and use the inequality $\left(1-e^{-a}\right) \geq a\left(1-e^{-1}\right)$ for $a \leq 1$ to obtain

$$
\frac{e}{e-1} \max \left\{1, \frac{\ell}{T}\right\}\left(1-e^{-T / \ell}\right) \geq \frac{e}{e-1} \frac{\ell}{T} \frac{T}{\ell}\left(1-e^{-1}\right)=1 .
$$

We are now able to express, for a key $x$, a dominating distribution to the distribution of the $k$ th smallest seed in $X \backslash x$ when using fixed $k \mathrm{SH}_{\ell}$.

Lemma E.2. The distribution $B$ of the $k$ th smallest seed, where seeds for $z \in \mathcal{X} \backslash x$ are independently drawn from $b_{\ell, w_{z}}$, is dominated by the function

$$
\frac{e}{e-1} \max \left\{1, \frac{\ell}{T}\right\} s_{W, k},
$$

where

$$
W=\sum_{z \in \mathcal{X}} \min \left\{w_{z}, T\right\}
$$

and $s_{W, k}$ is the density of $\operatorname{Erlang}(W, k)$. 
Proof. From Lemma E.1, $B$ is dominated by $\frac{e}{e-1} \max \{1, \ell / T\}$ times the density of the $k$ th seed according to $\operatorname{Exp}\left[\min \left\{w_{x}, T\right\}\right]$. The latter distribution is dominated by $s_{W, k}$ (using Lemma C.1). We get the claim from transitivity of domination.

\section{E.1 CV Bound for two-pass Estimator}

We are now ready to bound the variance of the fixed- $k \mathrm{SH}_{\ell}$ estimator when exact frequencies of sampled keys are available. The estimator is applied to an $\mathrm{SH}_{\ell}$ sample that includes the exact $\operatorname{cap}_{T}\left(w_{x}\right)$ values of sampled keys

We first bound the variance of the estimate for a key when conditioned on $\tau$.

LEMMA E.3.

$$
\operatorname{var}\left[\operatorname{cap} p_{T}\left(w_{x}\right) \mid \tau\right] \leq \max \left\{\frac{T}{\ell}, 1\right\} \frac{\min \left\{w_{x}, T\right\}}{\tau} .
$$

Proof. The inclusion probability of a key $x$ conditioned on the threshold $\tau$ is

$$
\operatorname{Pr}[\operatorname{seed}(x)<\tau]=\left\{\begin{array}{l}
\tau \ell>1:\left(1-e^{-\tau w_{x}}\right) \\
\tau \ell \leq 1:\left(1-e^{-\ell \tau}\right) \frac{1-e^{-w_{x} / \ell}}{1-1 / e} .
\end{array}\right.
$$

The variance conditioned on $\tau$ of the inverse probability estimate is

$$
\operatorname{var}\left[\operatorname{câp}_{T}\left(w_{x}\right) \mid \tau\right]=\left(\frac{1}{\operatorname{Pr}[\operatorname{seed}(x)<\tau]}-1\right) \min \left\{w_{x}, T\right\}^{2} .
$$

For $\tau \ell>1$ we have

$$
\begin{aligned}
\left(\frac{1}{\operatorname{Pr}[\operatorname{seed}(x)<\tau]}-1\right) & \leq \frac{e^{-\tau w_{x}}}{1-e^{-\tau w_{x}}} \\
& =\frac{1}{e^{\tau w_{x}}-1} \leq \frac{1}{\tau w_{x}} .
\end{aligned}
$$

The last inequality uses the relation $e^{x} \geq 1+x$ for $x \geq 0$. Substituting in Equation (30) we obtain

$$
\operatorname{var}\left[\operatorname{câp}_{T}\left(w_{x}\right) \mid \tau\right] \leq \min \left\{w_{x}, T\right\}^{2} \frac{1}{\tau w_{x}} \leq \frac{\min \left\{w_{x}, T\right\}}{\tau} .
$$

It remains to treat the case $\tau \ell \leq 1$. We will establish that

$$
\frac{1-1 / e}{\left(1-e^{-\ell \tau}\right)\left(1-e^{-w / \ell}\right)}-1 \leq \frac{1}{\min \left\{w_{x}, \ell\right\} \tau} \text {. }
$$

We first consider $w \geq \ell$. In this case the right-hand size is fixed at $1 /(\ell \tau)$. To maximize the lefthand size, over $w \geq \ell$, we take $w=\ell$. We then obtain that the left-hand size is at most $\frac{e^{-\ell \tau}}{1-e^{-\ell \tau}} \leq \frac{1}{\ell \tau}$, which establishes Equation (31).

We next consider $w \leq \ell$, recalling that we already assume $\tau \ell<1$, and thus have $w<\ell<1 / \tau$. To maximize the left-hand side of Equation (31) under these assumptions we need to minimize the denominator $h(\ell) \equiv\left(1-e^{-\ell \tau}\right)\left(1-e^{-w / \ell}\right)$ in the range $w<\ell<1 / \tau$. By taking the derivative $\frac{\partial h}{\partial \ell} \geq 0$, we see that it is negative in this range. Therefore, $h(\ell)$ is minimized for $\ell=1 / \tau$. Substituting $\ell=1 / \tau$, we obtain that the left-hand size of Equation (31) is at most $\frac{e^{-w \tau}}{1-e^{-w \tau}} \leq \frac{1}{w \tau}$, and thus Equation (31) is fully established. 
We now note that the left-hand size of Equation (31) is equal to $\frac{1}{\operatorname{Pr}[\operatorname{seed}(x)<\tau]}-1$. Substituting in Equation (30), we obtain that

$$
\begin{aligned}
\operatorname{var} & {\left[\operatorname{câp}_{T}(w) \mid \tau\right] } \\
& =\left(\frac{1}{\operatorname{Pr}[\operatorname{seed}(x)<\tau]}-1\right) \min \{w, T\}^{2} \\
& \leq \frac{1}{\ell \tau} \min \{w, T\}^{2} \\
& \leq \min \left\{\frac{w}{\ell}, \frac{T}{\ell}\right\} \frac{\min \{w, T\}}{\tau} \\
& \leq \min \left\{1, \frac{T}{\ell}\right\} \frac{\min \{w, T\}}{\tau} .
\end{aligned}
$$

The second-to-last inequality uses our assumption that $w \leq \ell$.

We are now ready to conclude the proof of Theorem 8.3. We bound the variance with respect to the distribution $B$ using the dominating function as in Lemma E.2. We obtain that the variance for key $x$ is

$$
\operatorname{var}\left[\operatorname{câp}_{T}\left(w_{x}\right)\right] \leq \frac{e}{e-1} \max \left\{\frac{T}{\ell}, \frac{\ell}{T}\right\} W \frac{\min \left\{w_{x}, T\right\}}{k-1} .
$$

We conclude as in the proof of Theorem C.3, showing that our estimate of $Q\left(\operatorname{cap}_{T}, H\right)$ for a segment $H$ with proportion $q$ of the $\operatorname{cap}_{T}$ statistics has CV that is at most $\left(\frac{e}{e-1} \frac{\max \{T / \ell, \ell / T\}}{q(k-1)}\right)^{0.5}$.

\section{E.2 CV Bound for One-Pass}

We provide the proof of Theorem 8.6, which bounds the variance of the one-pass estimators.

The estimators are applied to the same sample distribution of included keys and the proof outline is similar to that of the two-pass estimator (Theorem 8.3). The only component we are missing is a bound on the conditional variance $\operatorname{var}\left[\operatorname{câp}_{T}\left(w_{x}\right) \mid \tau\right]$ : Since the exact frequency $w_{x}$ is not available, we cannot apply Lemma E.3 and instead compute the variance of the one-pass estimator that is applied to the partial frequency count $c_{x}$.

LEMMA E.4.

$$
\begin{aligned}
\operatorname{var}\left[\operatorname{câp}_{T}\left(w_{x}\right) \mid \tau\right] & \leq \frac{\min \left\{w_{x}, T\right\}}{\tau}\left(\frac{\ell}{T}\left(1-e^{-T / \ell}\right)+\frac{T}{\ell}\right) \\
& \leq\left(1+\frac{T}{\ell}\right) \frac{\min \left\{w_{x}, T\right\}}{\tau} .
\end{aligned}
$$

Proof. The estimation coefficients $\beta(c)$ are provided in Theorem 8.5. We bound

$$
\begin{aligned}
\operatorname{var}\left[\operatorname{câp}_{T}(w) \mid \tau\right] & =\mathrm{E}\left[\beta(c)^{2}\right]-\mathrm{E}[\beta(c)]^{2} \\
& =\mathrm{E}\left[\beta(c)^{2}\right]-\min \{T, w\}^{2} .
\end{aligned}
$$

The last inequality follows from unbiasedness $\mathrm{E}[\beta(c)]=\operatorname{cap}_{T}(w)$.

For $\operatorname{cap}_{T}$, we have $f^{\prime}(x)=1$ for $x \leq T$ and $f^{\prime}(x)=0$ otherwise. Therefore,

$$
\beta(c)=\frac{\min \{T, c\}}{\min \{1, \ell \tau\}}+\tau^{-1} I_{c<T} .
$$

We use the density function of $c$ given $w$, which is provided in Theorem 8.4. The density function for $w \geq c>0$ is $\tau e^{-\tau(w-c)}$ when $\tau \ell \geq 1$ and $\tau e^{-(w-c) / \ell}$ when $\tau \ell \leq 1$. We have that $\beta(c)=0$ when $c=0$. We now use case analysis. 
We first consider the case $\tau \ell>1$ and $w \leq T$. We have $\beta(c)=c+1 / \tau$.

$$
\begin{aligned}
\mathrm{E}\left[\beta^{2}\right]-w^{2} & =-w^{2}+\int_{0}^{w} \tau e^{-\tau x} \beta(w-x)^{2} d x \\
& =-w^{2}+\int_{0}^{w} \tau e^{-\tau x}\left(\tau^{-1}+w-x\right)^{2} d x \\
& =\left(1-e^{-\tau w}\right)\left(w^{2}+\tau^{-2}\right)-w^{2} \leq w / \tau .
\end{aligned}
$$

The last inequality uses the relation $\left(1-e^{-x}\right) \leq x$.

For $\tau \ell>1$ and $w>T$, we have $\beta(c)=T$ for $w \geq c \geq T$ and $\beta(c)=c+1 / \tau$ for $c<T$. Therefore,

$$
\begin{aligned}
& \mathrm{E}\left[\beta^{2}\right]-T^{2} \\
& \quad=-T^{2}+\int_{0}^{w-T} \tau e^{-\tau x} T^{2} d x+\int_{w-T}^{w} \tau e^{-\tau x}\left(\tau^{-1}+w-x\right)^{2} d x \\
& \left.\quad=-T^{2}+\left(1-e^{-\tau(w-T)}\right) T^{2}+e^{-\tau(w-T)}\left(T^{2}+\tau^{-2}\right)-\tau^{-2} e^{-\tau w}\right) \\
& \quad=\tau^{-2} e^{-\tau w}\left(e^{\tau T}-1\right) \leq \tau^{-2}\left(1-e^{-\tau T}\right) \leq T / \tau .
\end{aligned}
$$

Using the fact that $e^{-T w}$ is maximized (subject to $w \geq T$ ) when $w=T$ and $\left(1-e^{-x}\right) \leq x$.

For $\tau \ell<1$ and $w \leq T$, we have $\beta(c)=\tau^{-1}(c / \ell+1)$.

$$
\begin{aligned}
\mathrm{E}\left[\beta^{2}\right] & =\int_{0}^{w} \tau e^{-x / \ell} \tau^{-2}\left(\frac{w+\ell-x}{\ell}\right)^{2} d x \\
& =\frac{1}{\tau \ell^{2}} \int_{0}^{w} e^{-x / \ell}(w+\ell-x)^{2} d x \\
& =\frac{\ell}{\tau}\left(1-e^{-w / \ell}\right)+\frac{w^{2}}{\tau \ell} \\
& =\frac{w}{\tau}\left(\frac{\ell}{w}\left(1-e^{-w / \ell}\right)+\frac{w}{\ell}\right) \\
& \leq \frac{w}{\tau}\left(\frac{\ell}{T}\left(1-e^{-T / \ell}\right)+\frac{T}{\ell}\right) \\
& \leq \frac{w}{\tau}\left(1+\frac{T}{\ell}\right) .
\end{aligned}
$$

The second to last inequality follows from the function $\left(1-e^{-x}\right) / x+x$ being increasing in the range $x>0$. Therefore, subject to fixed $\ell$ and $w \leq T$, the expression using $x=w / \ell$ is maximized at $w=T$.

For $\tau \ell<1$ and $w>T$ :

$$
\begin{aligned}
\mathrm{E}\left[\beta^{2}\right]= & \int_{0}^{w-T} \tau e^{-x / \ell} T^{2} /(\tau \ell)^{2} d x \\
& +\int_{w-T}^{w} \tau e^{-x / \ell}\left(\tau^{-1}+(w-x) /(\tau \ell)\right)^{2} d x \\
= & (\tau \ell)^{-1} T^{2}\left(1-e^{-(w-T) / \ell}\right) \\
& +(\tau \ell)^{-1} \int_{w-T}^{w}(1 / \ell) e^{-x / \ell}(\ell+w-x)^{2} d x
\end{aligned}
$$




$$
\begin{aligned}
= & (\tau \ell)^{-1}\left(T^{2}\left(1-e^{-(w-T) / \ell}\right)\right. \\
& \left.+e^{-(w-T) / \ell}\left(\ell^{2}+T^{2}\right)-\ell^{2} e^{-w / \ell}\right) \\
= & (\tau \ell)^{-1}\left(T^{2}+e^{-(w-T) / \ell} \ell^{2}-\ell^{2} e^{-w / \ell}\right) \\
= & \frac{T}{\tau}\left(\frac{T}{\ell}+\frac{\ell}{T} e^{-w / \ell}\left(e^{T / \ell}-1\right)\right) \\
\leq & \frac{T}{\tau}\left(\frac{T}{\ell}+\frac{\ell}{T}\left(1-e^{-T / \ell}\right)\right) \\
\leq & \frac{T}{\tau}(1+T / \ell) .
\end{aligned}
$$

The second to last derivation substitutes $w=T$ for the $w$ value that maximizes the expression subject to $w \geq T$. We then use $\left(1-e^{-x}\right) \leq x$.

\section{F DISCRETIZED THRESHOLD SAMPLING}

A variation on the fixed- $k$ discrete $\mathrm{SH}_{\ell}$ sampling scheme that can be useful in practice is to limit the algorithm to work with a discrete set of thresholds (think $\tau=\alpha^{i}$ for some $\alpha<1$ ) (see Algorithm 7).

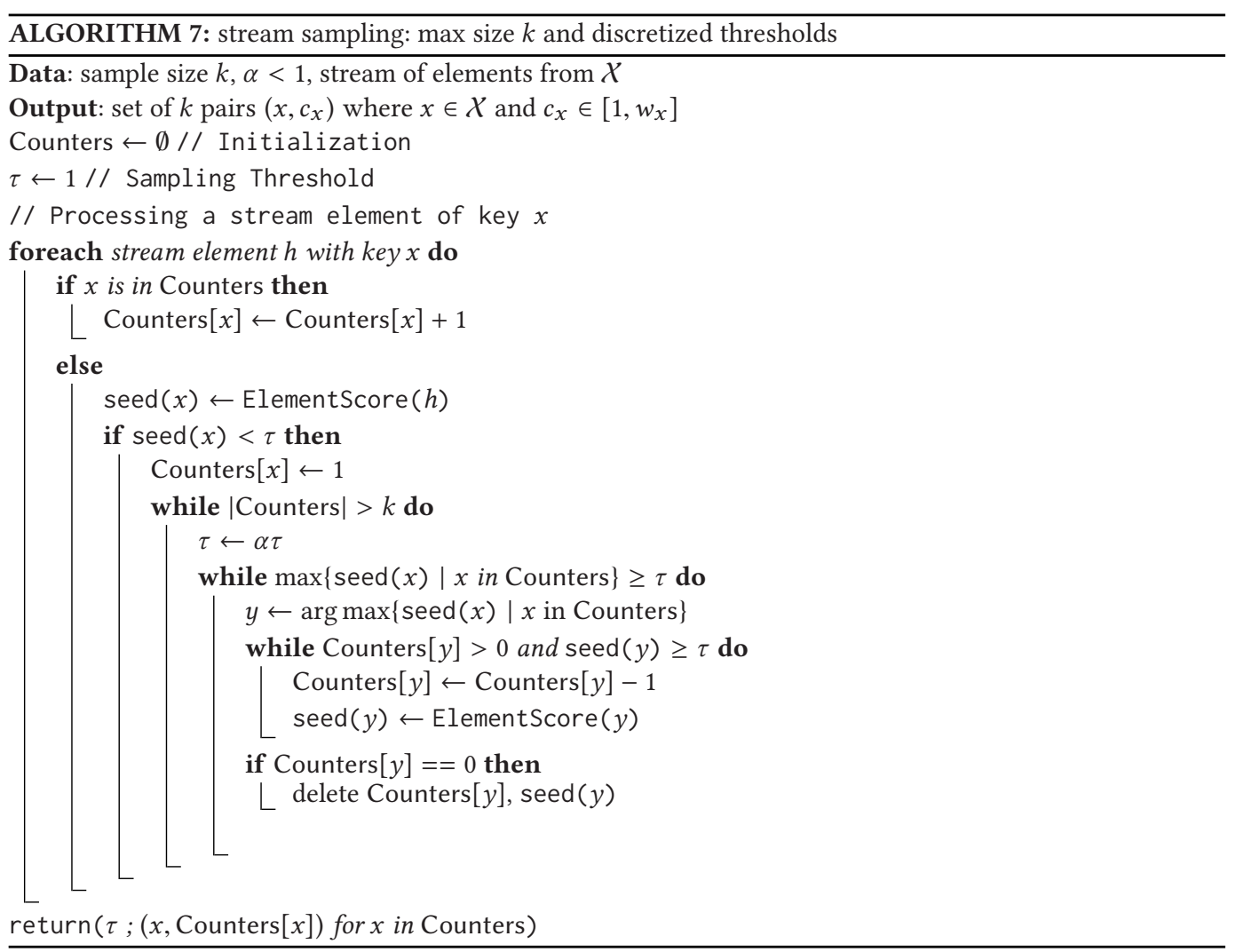


When the cache is full, the threshold is adjusted in iteration until its size drops below $k$. Discretized thresholds with fixed- $k$ SH were considered in Reference [12]. Discretized thresholds have the advantage that the number of times keys are pulled out/placed back on the priority queue for updates is lower. Another advantage is that the estimators, when expressed as coefficients that depend on $\ell$ and $\tau$, can be reused with different samples.

\section{G APPROXIMATE $\operatorname{cap}_{T}$ Counters}

Our design computes a sample of the active keys, over which segment statistics can be estimated. One can also consider the more basic problem of only estimating the statistics over the full dataset $Q(f, \mathcal{X})$.

The case $\operatorname{cap}_{1}$ corresponds to a (approximate) distinct count of keys, which is a fundamental and well-studied problem. The case cap $\mathrm{p}_{\infty}$ is the total frequency of the full stream that is trivially computed using a single counter.

Our constructions can be modified to be more efficient when we are only interested in estimating $Q\left(\operatorname{cap}_{T}, \mathcal{X}\right)$ : Instead of storing full identifiers of cached keys, which are needed for a sample, we can hash the key domain to a domain of size that is polynomial in the number $n$ of distinct keys. The resulting sketch size in this case would be $O(\log n)$ to represent each key hash and the count (which we can cap by a polynomial in $T$ to ensure the representation of the counts is at most $O(\log T)$. The result is an approximate $\operatorname{cap}_{T}$ counter on streams that has state (structure size) that is $O\left(\epsilon^{-2}(\log T+\log n)\right.$ and provides estimates with $\mathrm{CV}$ of $\epsilon$.

State-of-the-art approximate distinct counters, however, have a smaller, double logarithmic dependence on $n[20,25]$; in an appropriate representation, the state can be compressed to $O\left(\epsilon^{-2}+\log \log n\right)$. We present here a lightweight algorithm that provides a rough approximation of $Q\left(\operatorname{cap}_{T}, \mathcal{X}\right)$ with double logarithmic state. We apply to each stream element the string returned by the element scoring function ElementScore $(h)$ (7) used in our discrete $\mathrm{SH}_{T}$ algorithm. We then apply any off-the-shelf approximate distinct counter [6, 20, 21, 25] to the stream of ElementScore $(h)$. Recall that the elements being counted are the identifiers of key-bucket pairs from the original stream, where a bucket $b \sim U[1, \ldots, T]$ is drawn independently for each stream appearance of the key.

We now analyse the quality of this approximation.

Lemma G.1. The expected number of distinct strings generated is between $(1-1 / e) Q\left(\operatorname{cap}_{T}, \mathcal{X}\right)$ and $Q\left(\operatorname{cap}_{T}, \mathcal{X}\right)$.

Proof. The expected number of distinct strings that are generated for a key of cardinality $w$ is $\ell\left(1-(1-1 / T)^{w}\right)$. This is because the probability that we do not hit a certain bucket with $w$ elements is $(1-1 / T)^{w}$. Thus, the expected number of empty buckets is $T(1-1 / T)^{w}$.

So in expectation, a distinct counter applied with $T$ buckets would produce an underestimate. The worst relative error is obtained for keys with $w=T$, where the expected count is $T(1-1 / e)$, thus the relative error is $1 / e$. However, the error depends on the distribution of key sizes, and is small for cardinalities much larger or smaller than $T$.

This approach can obtain a rough estimate of $Q\left(\operatorname{cap}_{T}, \mathcal{X}\right)$ to within $(1-1 / e, 1)$ using state of size $O(\log \log n)$. (Since there is inherent error of $1-1 / e$, there is no benefit in using a more accurate distinct counter.)

One approach to reduce the error, left for future work, is to apply the counting with multiple values of $T$. 


\section{ACKNOWLEDGMENT}

The author thanks Kevin Lang for bringing to her attention the use of frequency capping in online advertising and the need for efficient sketches that support it. The author is grateful to the anonymous reviewers for a very thorough review that helped her improve the quality of the presentation.

\section{REFERENCES}

[1] N. Alon, Y. Matias, and M. Szegedy. 1999. The space complexity of approximating the frequency moments. f. Comput. System Sci. 58, 1 (1999), 137-147.

[2] V. Braverman and R. Ostrovsky. 2010. Zero-one frequency laws. In Proceedings of the Annual ACM Symposium on Theory of Computing Conference (STOC'10). ACM.

[3] K. R. W. Brewer, L. J. Early, and S. F. Joyce. 1972. Selecting several samples from a single population. Austr. J. Stat. 14, 3 (1972), 231-239.

[4] M. T. Chao. 1982. A general purpose unequal probability sampling plan. Biometrika 69, 3 (1982), 653-656.

[5] E. Cohen. 1997. Size-estimation framework with applications to transitive closure and reachability. F. Comput. System Sci. 55, 3 (1997), 441-453.

[6] E. Cohen. 2014. All-distances sketches, revisited: HIP estimators for massive graphs analysis. In Proceedings of the Pipeline Open Data Standard Conference (PODS'14). ACM.

[7] E. Cohen. 2015. Multi-objective weighted sampling. In Proceedings of the 2015 Third IEEE Workshop on Hot Topics in Web Systems and Technologies (HotWeb'15). IEEE.

[8] E. Cohen. 2015. Stream sampling for frequency cap statistics. In Proceedings of the Knowledge Discovery and Data Mining Conference (KDD'15). ACM, 2015.

[9] E. Cohen. 2017. Hyperloglog hyper extended: Sketches for concave sublinear frequency statistics. In Proceedings of the Knowledge Discovery and Data Mining Conference (KDD'17). ACM, 2017.

[10] E. Cohen, G. Cormode, and N. Duffield. 2012. Don't let the negatives bring you down: Sampling from streams of signed updates. In Proceedings of the ACM SIGMETRICS/Performance Conference.

[11] E. Cohen, N. Duffield, H. Kaplan, C. Lund, and M. Thorup. 2009. Composable, scalable, and accurate weight summarization of unaggregated datasets. Proc. VLDB 2, 1 (2009), 431-442.

[12] E. Cohen, N. Duffield, H. Kaplan, C. Lund, and M. Thorup. 2014. Algorithms and estimators for accurate summarization of unaggregated data streams. F. Comput. System Sci. 80, 7 (2014), 1214-1244. DOI : 10.1016/j.jcss.2014.04.009

[13] E. Cohen, N. Duffield, C. Lund, M. Thorup, and H. Kaplan. 2011. Efficient stream sampling for variance-optimal estimation of subset sums. SIAM 7. Comput. 40, 5 (2011).

[14] E. Cohen and H. Kaplan. 2008. Tighter estimation using bottom-k sketches. In Proceedings of the 34th VLDB Conference.

[15] E. Cohen, H. Kaplan, and S. Sen. 2009. Coordinated weighted sampling for estimating aggregates over multiple weight assignments. Proc. VLDB 2, 1-2 (2009).

[16] G. Cormode and S. Muthukrishnan. 2005. An improved data stream summary: The count-min sketch and its applications. F. Algor. 55, 1 (2005).

[17] N. Duffield, M. Thorup, and C. Lund. 2007. Priority sampling for estimating arbitrary subset sums. F. Assoc. Comput. Mach. 54, 6 (2007).

[18] C. Estan and G. Varghese. 2002. New directions in traffic measurement and accounting. In Proceedings of the Conference of the Special Interest Group on Data Communication (SIGCOMM'02). ACM.

[19] W. Feller. 1971. An Introduction to Probability Theory and Its Applications, Vol. 2. John Wiley \& Sons, New York, NY.

[20] P. Flajolet, E. Fusy, O. Gandouet, and F. Meunier. 2007. Hyperloglog: The analysis of a near-optimal cardinality estimation algorithm. In Analysis of Algorithms. DMTCS.

[21] P. Flajolet and G. N. Martin. 1985. Probabilistic counting algorithms for data base applications. f. Comput. System Sci. 31, 2 (1985), 182-209. https://doi.org/10.1016/0022-0000(85)90041-8.

[22] R. Gemulla, W. Lehner, and P. J. Haas. 2006. A dip in the reservoir: Maintaining sample synopses of evolving datasets. In Proceedings of the 32nd International Conference on Very Large Data Bases (VLDB'06). 595-606.

[23] P. Gibbons and Y. Matias. 1998. New sampling-based summary statistics for improving approximate query answers. In Proceedings of the Conference of the Special Interest Group on Management of Data (SIGMOD'98). ACM.

[24] Google. Frequency capping: AdWords help. Retrieved December 2014 from https://support.google.com/adwords/ answer/117579.

[25] S. Heule, M. Nunkesser, and A. Hall. 2013. HyperLogLog in practice: Algorithmic engineering of a state of the art cardinality estimation algorithm. In Proceedings of the International Conference on Extending Database Technology (EDBT'13).

[26] N. Hohn and D. Veitch. 2003. Inverting sampled traffic. In Proceedings of the 3rd ACM SIGCOMM Conference on Internet Measurement. 222-233. 
[27] D. G. Horvitz and D. J. Thompson. 1952. A generalization of sampling without replacement from a finite universe. 7 . Am. Stat. Assoc. 47, 260 (1952), 663-685.

[28] P. Indyk. 2001. Stable distributions, pseudorandom generators, embeddings and data stream computation. In Proceedings of the 41st IEEE Annual Symposium on Foundations of Computer Science. IEEE, 189-197.

[29] W. Johnson and J. Lindenstrauss. 1984. Extensions of Lipschitz mappings into a Hilbert space. Contemporary Math. 26.

[30] H. Jowhari, M. Saglam, and G. Tardos. 2011. Tight bounds for Lp samplers, finding duplicates in streams, and related problems. In Proceedings of the Pipeline Open Data Standard Conference (PODS'11).

[31] D. E. Knuth. 1968. The Art of Computer Programming, Vol. 2, Seminumerical Algorithms (1st ed.). Addison-Wesley.

[32] J. Misra and D. Gries. 1982. Finding repeated elements. Technical Report, Cornell University.

[33] M. Monemizadeh and D. P. Woodruff. 2010. one-pass relative-error $\mathrm{l}_{\mathrm{p}}$-sampling with applications. In Proceedings of the 21st ACM-SIAM Symposium on Discrete Algorithms. ACM-SIAM.

[34] E. Ohlsson. 1998. Sequential poisson sampling. J. Off. Stat. 14, 2 (1998), 149-162.

[35] M. Osborne. Facebook Reach and Frequency Buying. Retrieved October 2014 from http://citizennet.com/blog/2014/10/ 01/facebook-reach-and-frequency-buying/.

[36] B. Rosén. 1972. Asymptotic theory for successive sampling with varying probabilities without replacement, I. Ann. Math. Stat. 43, 2 (1972), 373-397.

[37] B. Rosén. 1997. Asymptotic theory for order sampling. F. Stat. Plan. Inf. 62, 2 (1997), 135-158.

[38] M. Szegedy. 2005. Near optimality of the priority sampling procedure. Technical Report TR05-001, Electronic Colloquium on Computational Complexity.

[39] Y. Tillé. 2006. Sampling Algorithms. Springer-Verlag, New York.

Received March 2017; revised December 2017; accepted June 2018 